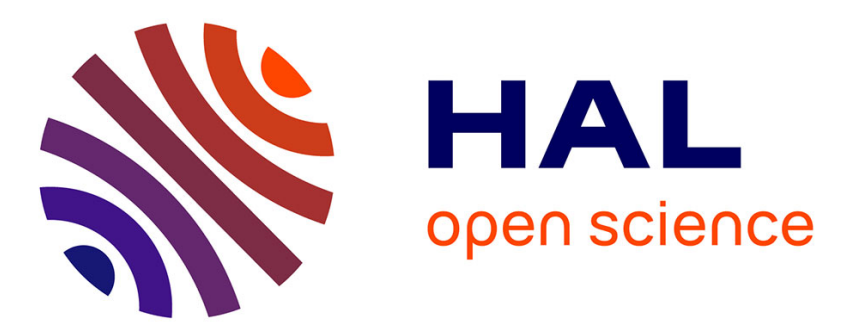

\title{
Quadri-joint inversion: Method and application to the Big Sky 9C 3D data set in northern Montana
}

Vincent Clochard, Bryan Devault, David Bowen, Nicolas Delépine, Kanokkarn Wangkawong

\section{- To cite this version:}

Vincent Clochard, Bryan Devault, David Bowen, Nicolas Delépine, Kanokkarn Wangkawong. Quadrijoint inversion: Method and application to the Big Sky 9C 3D data set in northern Montana. Interpretation, 2018, 6 (4), pp.SN101-SN118. 10.1190/INT-2018-0035.1 . hal-02014426

\section{HAL Id: hal-02014426 \\ https: / hal-ifp.archives-ouvertes.fr/hal-02014426}

Submitted on 11 Feb 2019

HAL is a multi-disciplinary open access archive for the deposit and dissemination of scientific research documents, whether they are published or not. The documents may come from teaching and research institutions in France or abroad, or from public or private research centers.
L'archive ouverte pluridisciplinaire HAL, est destinée au dépôt et à la diffusion de documents scientifiques de niveau recherche, publiés ou non, émanant des établissements d'enseignement et de recherche français ou étrangers, des laboratoires publics ou privés. 


\title{
Quadri-joint inversion: method and application to the Big Sky 9C 3D dataset in Northern Montana
}

\author{
Vincent Clochard (IFP Energies nouvelles) \\ Bryan C. DeVault (Vecta Oil \& Gas) \\ David Bowen (Vecta Oil \& Gas) \\ Nicolas Delépine (IFP Energies nouvelles) \\ Kanokkarn Wangkawong (IFP Energies nouvelles, currently PTTEP).
}

\begin{abstract}
The Big Sky Carbon Sequestration Partnership is a $\mathrm{US} \mathrm{CO}_{2}$ storage project in the Duperow formation from the Kevin Dome structure located in Montana. The goal is to characterize the Duperow interval as a potential long-term storage zone for injected $\mathrm{CO}_{2}$. During the years 2013-2015, a North American company, Vecta Oil \& Gas operated a multi-component (9C) seismic survey on behalf of the Partnership over the Kevin Dome, using $P$ - and $S$-wave sources.
\end{abstract}

After processing of 9C 3D seismic data, pre-stack $P P, P S, S H$ and $S V$ datasets were generated and migrated in their corresponding time domains. We evaluate from the real case study the benefits of inverting jointly $P P, P S, S H$ and $S V$ pre-stack seismic data. We compute different registration laws like $P P-P S, P P-S H$ and $P P-S V$ using a warping (time shift computation) procedure based on inverted shear-wave impedances from sequential inversions. This parameter choice represents a significant improvement over methods which rely on attempting to match trace waveforms which may have different phase, frequency content, and polarity.

The presence of Bakken formation just above the Duperow formation allow us to evaluate also the benefits of inverting jointly $P P, P S, S H$ and $S V$ pre-stack seismic data in recovering the elastic rock properties, like the inverted density parameter. As a matter of fact, the exploitation of unconventional hydrocarbons requires innovative skills to allow better characterization of brittle reservoir zone. The Young's modulus is a measure of their brittleness and requires an accurate determination of the rock density which is well known only at the well locations.

This real case study of multi-component (9C) pre-stack stratigraphic inversion is an elegant way of employing all modes of a multi-component acquisition to generate an optimal estimate of $P$ - and $S$ wave impedances and density to determine lithology and fluid contents further used by quantitative reservoir characterization. 


\section{Introduction}

The Big Sky Carbon Sequestration Partnership (BSCSP) is a US $\mathrm{CO}_{2}$ storage project in which the main target is a porous dolomite layer within the Duperow formation located on the Kevin Dome structure (bigskyco2.org), a large anticline in Toole County, Montana. The main goals of the project were to safely sequester $\mathrm{CO}_{2}$ into the Middle Duperow and verify that the caprock overlaying the Duperow formation is an impermeable geologic formation able to contain over the long term 1 million tons of $\mathrm{CO}_{2}$. Part of the project included the acquisition of a $50+\mathrm{km}^{2} 9 \mathrm{C}-3 \mathrm{D}$ survey that employed both $P$ - and $S$-wave sources. After pre-processing and limited angle stack imaging of the 9C-3D seismic data (Murray et al, 2016), a full series of pre-stack PP, PS, SH and $S V$ datasets (Near, Mid, Far angle stacks) were generated and migrated in their corresponding time domain.

It has long been known from published sensitivity analyses that $P P$ mono-component inversion gives accurate $P$-wave impedance results but often imprecise $S$-wave impedance and always irrelevant density results, as confirmed by numerous theoretical studies (Hilterman, 2001, Swan, 1993, Lebrun et al., 2001, Garotta et al., 2002). Previous inversion case studies have used subsets of the full multicomponent wavefield like PP-PS from OBS/OBC offshore datasets (Side et al., 2000, Garotta et al., 2000, Dariu et al., 2003, Barnola and Ibram, 2014) or PP-SH data from onshore datasets (DeVault et al., 2007) and more recently with PP-prestack and SS-poststack datasets (Butler et al., 2016). The goal of our study is to evaluate with a real case study the benefits of inverting jointly a much fuller wavefield comprising $P P, P S, S H$ and $S V$ prestack seismic data.

In the context of unconventional hydrocarbons, the presence of Bakken and Banff formations just above the Duperow formation allows us to evaluate the benefits of inverting jointly $P P, P S, S H$ and $S V$ pre-stack seismic data to recover the elastic rock properties like the inverted density parameter which is a key parameter required to compute brittleness in order to better characterize "sweet spots" which are linked to brittle rocks (Chopra and Sharma, 2015 and more recently Butler et al., 2016). Limited to pre-stack inversion of the $P P$ wavefield only, the problem of finding accurate attribute-combination properties depending on $P$ - and $S$-wave impedances like $\lambda \rho, \mu \rho$ or Young's modulus multiplied by density $(E \rho)$ (Sharma and Chopra, 2015a) remains extremely challenging. Also due to the limited seismic bandwidth, characterization of thin sweet spots remains challenging (Sharma and Chopra, $2015 \mathrm{~b}$ ). For all of these reasons, accurate recovery of the density parameter through the inversion process could greatly improve the quality of seismically-derived brittleness estimates for resource play sweet spot identification.
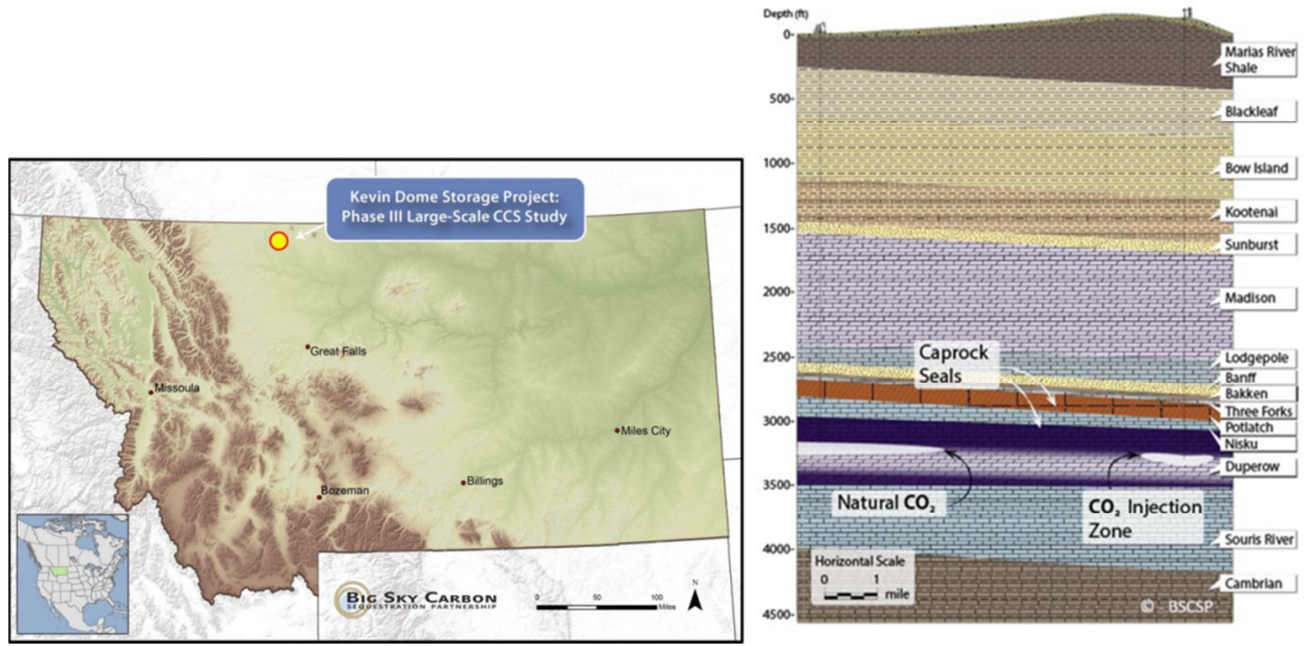

Figure 1: The BIG SKY $\mathrm{CO}_{2}$ storage project in Montana, US. Kevin Dome is a potential structure and target at porous Dolomite formation, Duperow. 


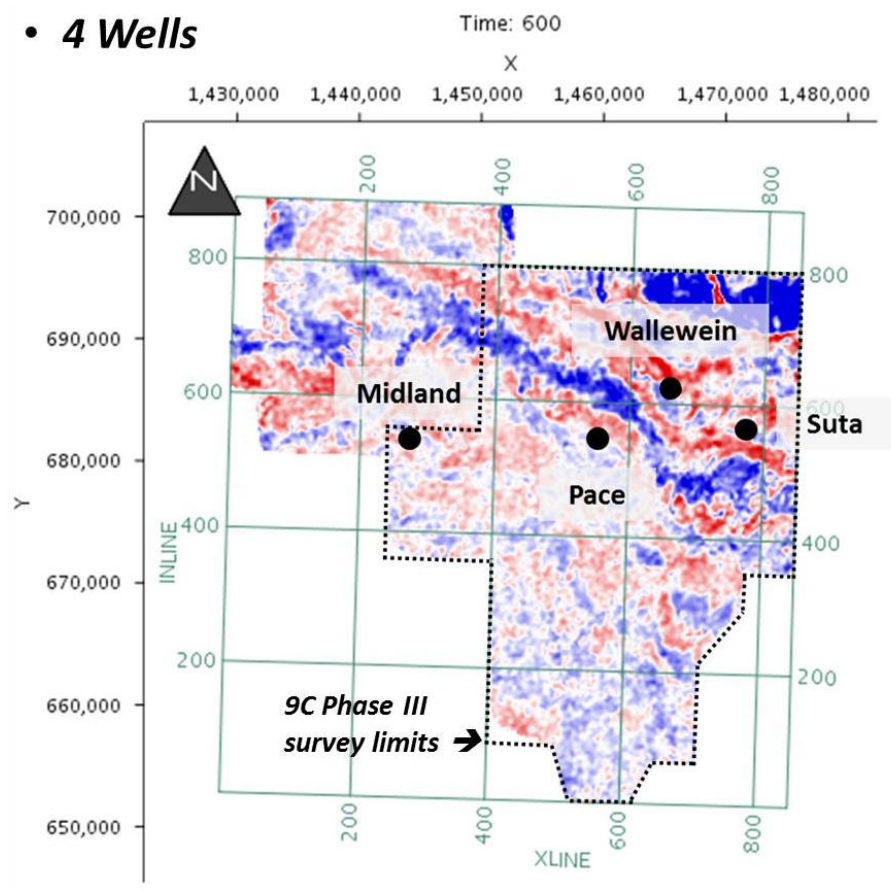

Figure 2: Seismic survey recoded during Phase III of The BIG SKY $\mathrm{CO}_{2}$ storage project. One can note that the PP wavefield coverage is more extensive, in the north-west, than that of the multicomponent wavefields.

\section{Quadri-joint inversion: a novel technique for integrating the information in 9C prestack seismic data}

\section{The joint multicomponent inversion workflow}

We have developed a three-step multicomponent inversion workflow able to jointly invert all kinds of seismic wavefields including $P P, P S, S V$ and $S H$. This workflow provides a unique optimal earth model in $P P$ two way time $\left(\mathrm{TWT}^{P P}\right.$ ). The earth model is parameterized by three isotropic elastic parameters which are $P$ - and $S$-wave impedances (IP and $I S)$ and density $(\rho)$ distributions. In the following section we describe the three-step multicomponent inversion workflow:

- $\underline{\text { Step-1: }}$ wavefield-independent pre-stack stratigraphic inversion (Tonellot et al., 2001) of a given wavefield $P P, P S, S V$ or $S H$ characterized by 3 migrated angle stacks. Inversions are independent from each other and they provide an optimal model recorded in the $T W T$ domain of the corresponding wavefield. Any kind of inversion provides an optimal earth model parameterized by three elastic parameters: $P$ - and $S$-wave impedances and density $(I P, I S, \rho)$. The inversion procedure employed here uses the following linearization of the Zoeppritz (1919) isotropic reflection coefficients: $P P$-reflectivity from Aki and Richards (1980); $P S$-reflectivity given by Ramos and Castagna (2001); and $S H$ - and $S V$-reflectivities derived by Rüger (1996). One can note that the resulting signal to noise ratio differs among the estimated quantities depending on which mode or combination of modes, angle stacks are inverted: for example with $P P$-inversion $(I P, I S, \rho), \rho$ should never be used for reservoir characterization. Inversion using additional $P S$ wavefield will better recover $I S$ and $\rho$ compare to $P P$-inversion although the density estimate for that mode is generally of very low quality. Same remark using additional $S H$ - or $S V$-wavefield which produce also a better contribution with density inverted parameter (Lebrun et al., 2001). 


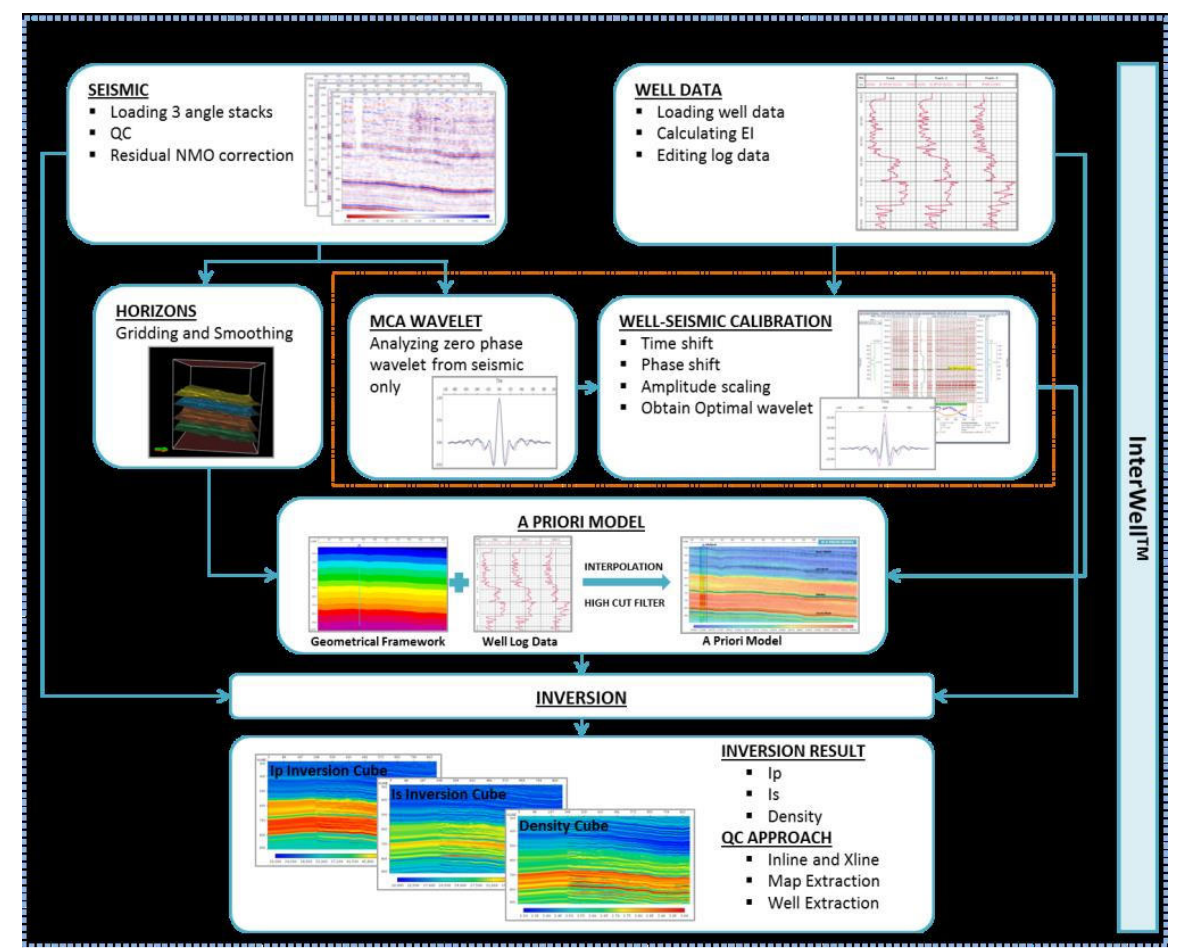

Figure 3: Description of the pre-stack inversion workflow applied to a given wavefield using 3 angle stacks. It is applied independently for all available wavefields using their migrated angle stacks. In the following case study we use PP $\left[0^{\circ}-15^{\circ}\right],\left[15^{\circ}-30^{\circ}\right],\left[30^{\circ}-45^{\circ}\right], \boldsymbol{P S}\left[15^{\circ}-25^{\circ}\right],\left[25^{\circ}-\right.$ $\left.35^{\circ}\right],\left[35^{\circ}-45^{\circ}\right]$, SH $\left[0^{\circ}-10^{\circ}\right],\left[10^{\circ}-20^{\circ}\right],\left[20^{\circ}-30^{\circ}\right]$ and $\boldsymbol{S V}\left[0^{\circ}-7^{\circ}\right],\left[7^{\circ}-14^{\circ}\right],\left[14^{\circ}-21^{\circ}\right]$

- $\underline{\text { Step-2: }}$ computation of registration law (warping). The goal of this step is to find a registration law connecting the $P P$ and multicomponent seismic time scales based on a time shift analysis considering $\boldsymbol{P P}$ and $\boldsymbol{P S}, \boldsymbol{S H}$, and $\boldsymbol{S} \boldsymbol{V}$ seismic events. Agullo et al. (2004) considered the optimal $\boldsymbol{S}$ wave impedances $(I S)$ from each sequential inversion, instead of working from seismic amplitudes including seismic noise. This solution has the advantage that it compares like physical quantities across the various modes while implicitly resolving wavelet phase and frequency issues through the inversion process. The problem is parameterized by starting with an initial registration law described below. Using the $S$-wave impedance suitably warped with the starting model, we determined the optimal time shift with a 3D-semblance algorithm based on the measurement of square difference (local $L^{2}$ norm) of two impedance cubes according to a series of time shifts applied to the multicomponent one (see parameter description from Table 1). A problem appears when impedances from the two cubes are not similar so that the minimization could fall into a local minimum. We have found a solution taking into account a threshold applied to a quality factor of the semblance, the resulting "holes" in the cube of time shifts are then filled by an iterative interpolation starting from its edge toward its interior, so that bad time shifts are iteratively replaced by the average time shift of the neighboring point (already interpolated or not). The iterative procedure will end when all "holes" are fully filled. 


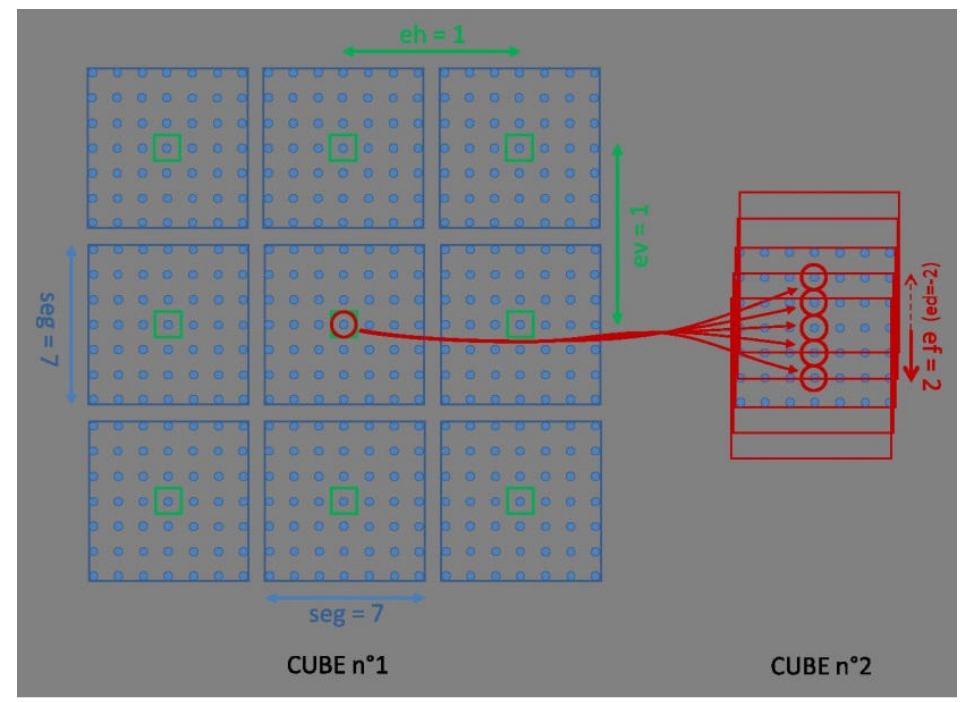

Table 1: Main parameters of the time shift computation between two cubes. From cube 1 we display the initial pixels in blue color; cube 2 must have the same dimensions (identical number of pixels in 3 dimensions). Red squares from cube 2 represent the moving windows to find the optimal time shift from a given pixel of the decimated cube 1(denoted by a green square). Maximum time shift $\boldsymbol{e f}$ (positive like TWT) and $\boldsymbol{e d}$ (opposite of $\boldsymbol{e f}$ ) are given by their respective number of samples; this is at least equal to 2 because it requires 5 points (center and 2 neighbors on each side, points inside the red circles) to compute the optimal time shift (done by a minimum of the parabola algorithm). Decimation rate of the original cube given by seg parameter (at least 1 and equal to 7 on the example) may be used to reduce memory requirements. We can also apply a slight vertical and horizontal smoothing to $\boldsymbol{e h}$ and $\boldsymbol{e v}$ which are small because they are applied on decimated data (points inside the green squares).

We illustrate the warping procedure considering only $P P$ and $P S$ inversions (result of step 1), giving a registration law to convert seismic event from $\mathrm{TWT}^{P P}$ to $\mathrm{TWT}^{P S}$. Using a synthetic $P P-P S$ seismic dataset (Agullo et al., 2004) Figure 4 shows the warping computation with $P P$ and $P S$ wavefields:

(1) Global constant vertical stretch of inverted $S$-wave impedance in $\mathrm{TWT}^{P P}, I S^{P P}$ gives $I S$ in $X P P$ time, $\boldsymbol{I} \boldsymbol{S}^{X P P}$. This process uses the same $\mathrm{Vp} / \mathrm{Vs}$ ratio all along the time axis.

(2) Local refinement of time shift based on a semblance algorithm described above between the $\boldsymbol{I} \boldsymbol{S}^{X P P}$ and inverted $S$-wave impedance from $P S$ wavefield $\left(\boldsymbol{I} \boldsymbol{S}^{P S}\right)$. The procedure is similar considering $P P-S H$ and $P P-S V$ wavefields and finally by the same way we have computed the $P P$ $S H$ and $P P-S V$ registration laws.

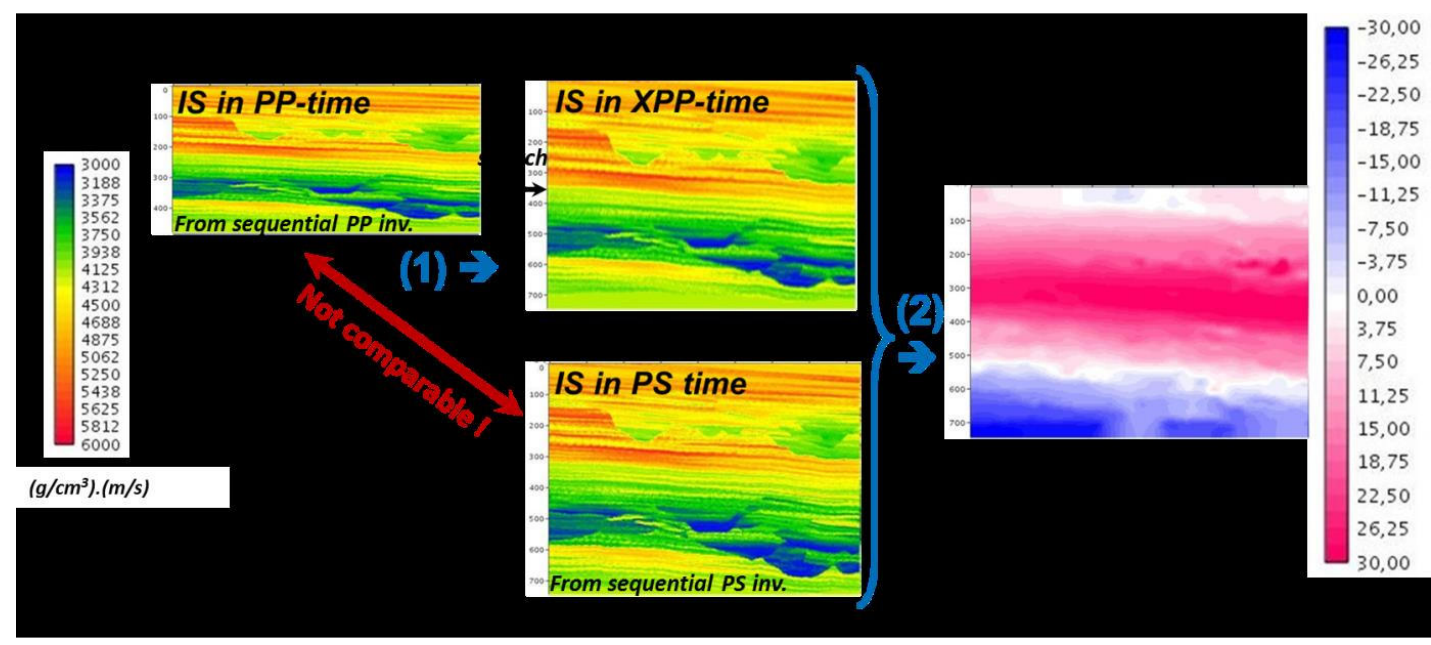


Figure 4: Example of a computation PP-PS time shift in order to build a scaling law to match PP and PS seismic events. Plot on the right is the result of the time shift refinement which has to be added to the constant vertical stretch supplied by the initial model.

- Step-3: Joint prestack stratigraphic inversion. The joint inversion simultaneously uses several kinds of wavefields. The seismic term of the cost function has terms for each input wavefield's migrated angle stacks. The joint inversion process always starts with a unique a priori model built in $P P$-time. As part of this case study we defined all possible joint inversions: Bi-joint as the $P P$ $P S, P P-S H$ or $P P-S V$ inversions using only one corresponding registration law from step 2; Trijoint $P P-S H-S V, P P-P S-S V$ or $P P-P S-S H$ inversions using two corresponding registration laws from step 2; and the final Quadri-joint $P P-P S-S H-S V$ inversion using the three registration laws from step 2. To achieve the final quadri-joint inversion the optimizer has to invert 12 (ie 4 wavefields by 3 angle stacks) input seismic cubes in the whole inversion process which takes only half an hour with 64 processors and 20 iterations. The summary of inversion parameters is displayed in Table 2 and is a unique set of parameters used for all kinds of inversion in the following.

\begin{tabular}{lc}
\multicolumn{2}{c}{ Inversion parameters } \\
Sigma I $I_{p}\left(\sigma_{p}\right)$ & $10,000.0$ \\
Sigma I $I_{S}\left(\sigma_{S}\right)$ & $8,000.0$ \\
Sigma Density $\left(\sigma_{\rho}\right)$ & 0.5 \\
Lambda $X\left(L_{x}\right)$ & $1,100.0 \mathrm{ft}$. \\
Lambda $Y\left(L_{\mathrm{y}}\right)$ & $1,100.0 \mathrm{ft}$.
\end{tabular}

Table 2: Main parameters of the optimizer. They could be defined globally for a given interest area or for each each geological units of the interest area. The Sigma parameters are defined for the three elastic parameters and govern how far the optimizer has to go from the a priori model. The Lambda parameters is relative to correlation length. One has to keep in mind small Sigma will produce short lateral correlations on the inverted result, and large Sigma will produce large lateral correlations including more a priori geological knowledge.

\section{$\underline{\text { QC of sequential inversion (step 1) from Kevin Dome }}$}

After a pre-stack inversion, it is important to compare the inversion residuals with the observed angle stacks to judge the reasonableness of the inversion output and check for any systematic mismatches that might indicate problems with the wavelet, input angle stacks, or background model. The next step of the QC process is to compare the inversion results at wells inside the seismic dataset with their respective logs (suitably band limited to the seismic bandwidth), transformed to the Two Way Time domain. 


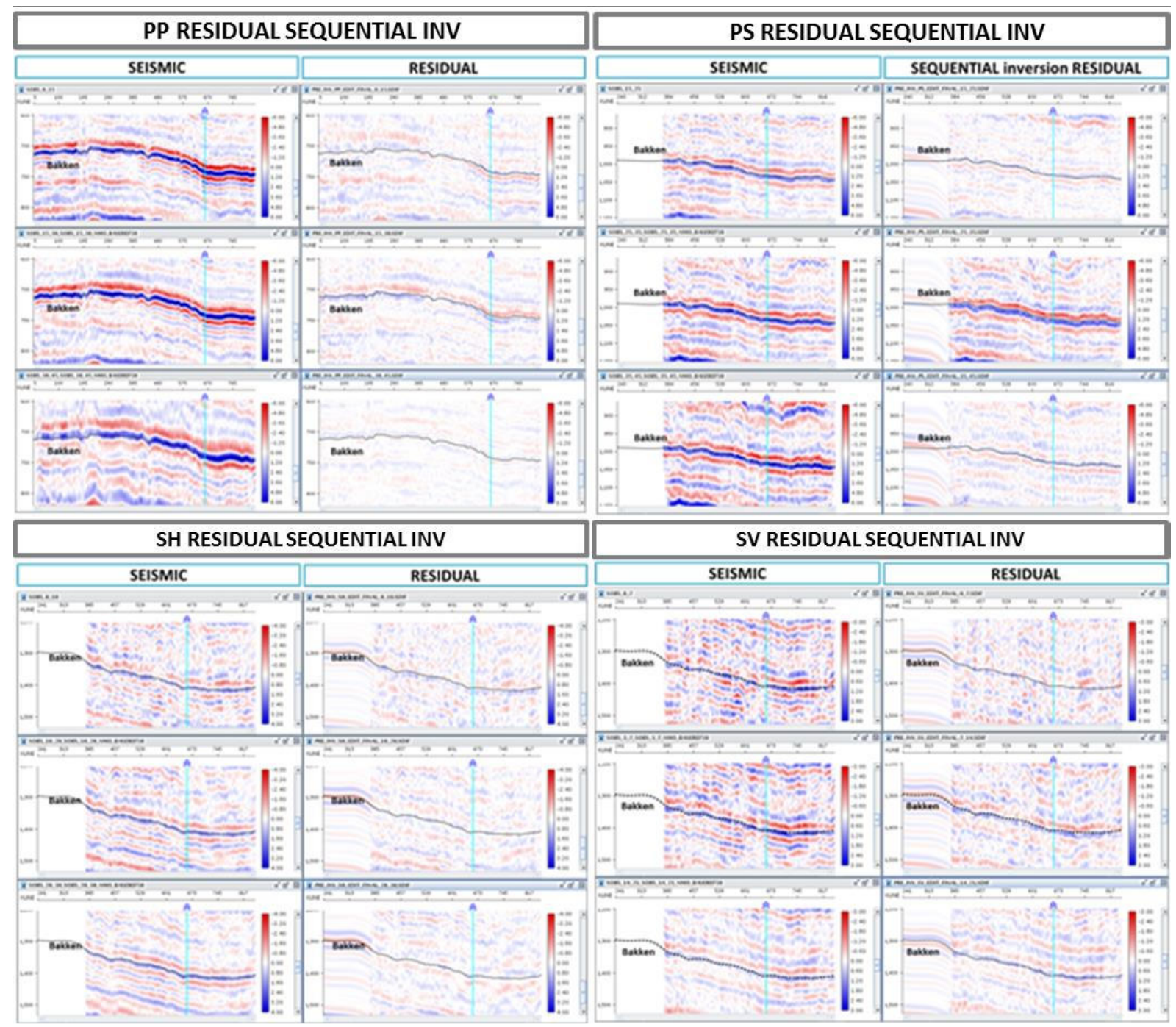

Figure 5: For each wavefield PP, PS, SH, SV comparison of the 3 input angle stacks (Near, Mid, Far) with residuals obtained after independent inversion of each wavefield. Vertical cyan line on the figure is at the location of the Wallewein 22-1 well drilled by the BSCSP. Here all residuals are weak except for the middle angle stack of the PS wavefield, the QC possibly indicating a minor issue with the scaling of the wavelet or seismic amplitude before construction of input angle stacks used by inversion.

Residuals from four sequential independent inversions are weak and they have a random distribution of seismic amplitudes (Figure 5). Nevertheless one can observe stronger residuals of the middle angle stack of the PS pre-stack inversion. The reason for this is presently unknown but may indicate a suboptimal relative amplitude preservation for this stack alone during pre-processing. Another possible explanation could be difficulties in the migration process but any such issues would also have affected the near and far angle stacks which is not the case because those residuals are weak.

Examination of the residuals alone is not enough to verify the quality of the inversion result: it is even more important to compare the inverted log (red curves on the following Figure 6) with the observed log (black curves) and a priori model logs (blue curves). For this comparison we have chosen the Wallewein 22-1 well (located in the middle of the survey to avoid eventual edge effects). It is interesting to see how the quality of the inversion result at the well is affected by the choice of wavefields chosen as inputs to the inversion (Figure 6). 
From the $P P$-inversion the inverted $I P$ and $I S$ (Figure 6 upper left) look quite accurate, but the density log has strong oscillation-like ringing artifacts especially in the shallow Cretaceous section above the Sunburst which is characterized by shales prone to washout and therefore unreliable log values, particularly for the pad-contact density tool during drilling operations. From the theoretical point of view retrieving the density parameter is not accurate enough with only the $P P$ wavefield, as described previously. Our interpretation of these oscillationlike ringing artifacts is that they are due to the combination of the insensitivity of even wideaperture $P P$ data to density changes with the large degree of freedom allowed by the value of uncertainty-density parameter $\left(\sigma_{\rho}=0.5\right)$. With the usual inversion input containing only the $P P$ wavefield, the user would have reduce $\sigma$ to small values close to 0.1 to avoid these oscillation-like ringing artifacts, but the goal of the study is to demonstrate that using multicomponent wavefields could improve inverted $I S$ and density parameter estimation without excessive a priori constraint of the inverted parameters through the uncertainty parameter given by $\sigma$ values. One has to keep in mind, they control how far the optimizer is allowed to deviate from the a priori model to find the best solution.

From the $S H$ - and $S V$-inverted logs (Figure 6 right column), $I P$ is not inverted for (see label "Cannot invert" displayed), due to the reflectivity formula not containing the $P$-wave impedance; the $I S$ inverted log follow more or less the trend of the measured (black curves) and a priori (blue curves) logs. Again, the density logs are ringing like the $P P$-inversion, from top to bottom of the images.

From the $P S$-inverted log (Figure 6, bottom left), it is clear that the density log is even more oscillation-like ringing than $S H$ and $S V$ inverted log, from the top to bottom of the image. 


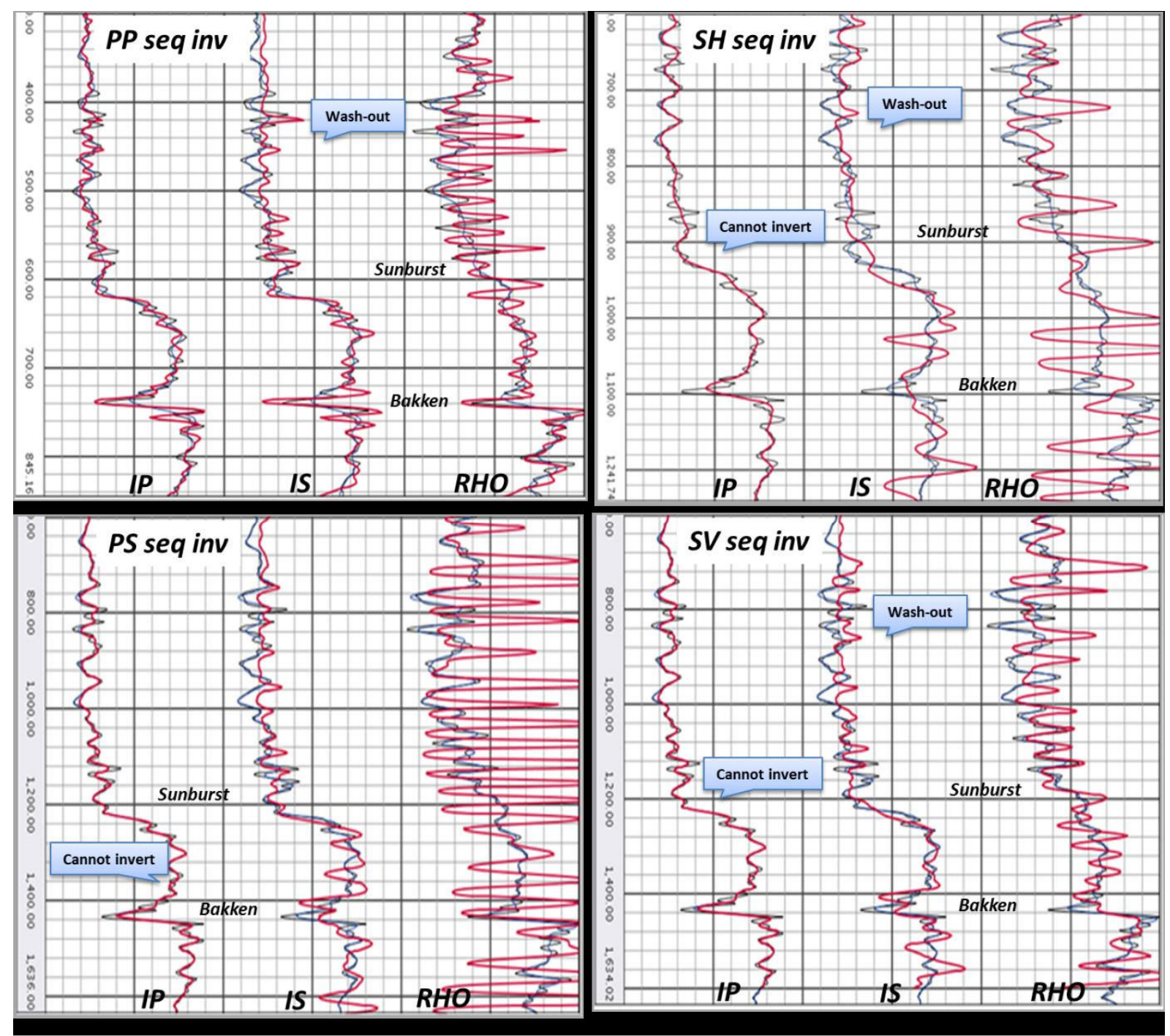

Figure 6: at Wallewein 22-1 well, inverted IP, IS, Density log from independent and single inversion of each wavefield (red curves). Log measurements (black curves) and a priori log, extracted from a priori model used by the inversion process (blue curves) are shown. Here the oscillation-like ringing artefacts, especially on the density, are due to our final choice of parameter for the quadri-joint inversion. There are too many degrees of freedom for the optimizer with only one wavefield to invert. In the following, we can see how these artefacts will decrease drastically through horizon maps (Figure 13 and 14) showing limited range of values.

In conclusion, sequential and independent pre-stack inversion of all the individual wavefields $(P P, P S, S H, S V)$ gives very good residuals because they have all random distribution with weak amplitudes compared to the observed seismic. Nevertheless the extracted inverted logs show significant differences at the well and one can observe strong oscillation-like ringing artefacts, particularly for the density estimate derived from each inversion.

It is important to note that the choice of inversion parameters for the sequential independent inversions are the same as those for the joint inversion described in more detail below. Looking at windowed extractions made on the three main targets (the Sunburst, Bakken/Banff and Duperow, Figure 7), we can observe in the right columns that the density parameter has values clearly outside of the range we will obtain with joint inversion. This is especially true with the Bakken/Banff formation where the map shows dark blue colors everywhere. More 
precisely the distribution of values is between 2.4 and 2.7 (see Figure 7, the right map with a different scale to see better the density distribution) which greatly exceeds the range of densities observed in the available well control within the survey. It is expected that shales and near-shore sand/siltstone units deposited in the relatively quiet setting of Bakken/Banff time should exhibit variations less than about 10\%. These results from the Step 1 workflow seem to be in agreement with the theoretical observation that PP pre-stack inversion is not able to recover properly the measured range of density values. (Lebrun et al., 2001, Garotta et al., 2002). From this observation we deduce the same conclusion for the two other targets at the Duperow (map showing saturated colors with dark blue and dark red) and Sunburst (map showing saturated dark red color).

Acquisition footprint (left and middle columns) appears only for $P$ - and $S$-wave impedances in the upper maps (Sunburst and Bakken). Deeper at the Duperow level they have completely disappeared with the same set of inversion parameters and they appear linked to the level of signal to noise ratio of recorded seismic. In practical terms, if only the $P P$ wavefield is available, we should adapt the inversion parameters set like reducing drastically the uncertainty parameter on the density $\left(\sigma_{\rho}=0.1\right)$, but this was not the purpose of our study.

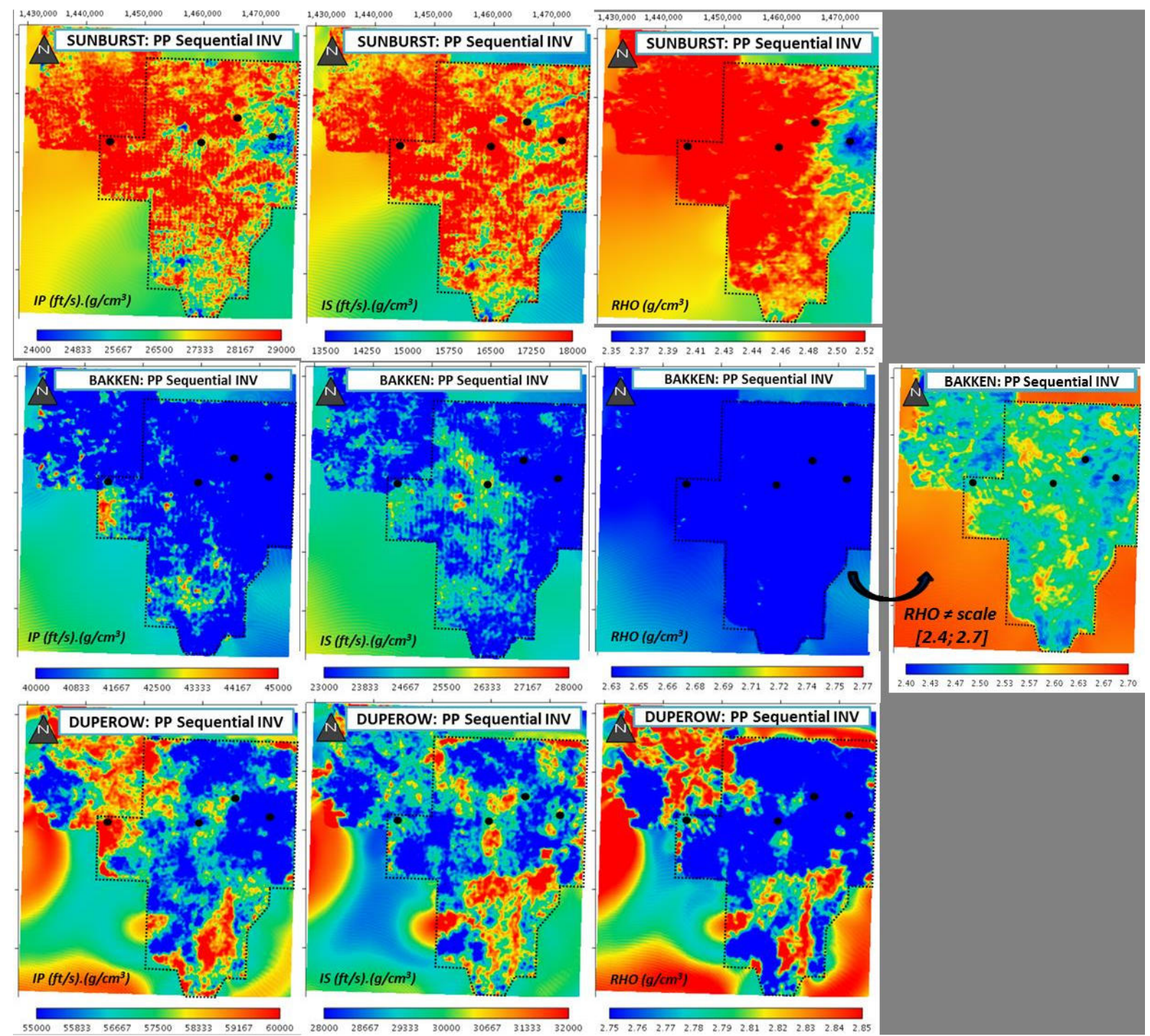

Figure 7: Display of PP-inversion results, considering the 3 main targets, the Sunburst, Bakken and Duperow. We notice strong oversaturation due to wide range of inverted density 
values (right column) especially with Bakken map where the geological context does not allow strong lateral variations like a density variation from 2.4 to $2.7 \mathrm{~g} / \mathrm{cc}$. Notice some strong acquisition footprints appearing with $P$ - and S-wave horizon maps at the Sunburst and Bakken (2 first rows). These are due to seismic noise which has been inverted and there is less noise in the deeper horizon maps (third row).

\section{Multicomponent warping results (step 2) from Kevin Dome}

After performing independent pre-stack inversions of each kind of wavefield (Step 1 of the multicomponent inversion workflow), 3D time shifts are computed from inverted $S$-wave impedance $(I S)$ as described in more detail in the description of step 2 above. In the following three figures, we have displayed time-shift results and QCs of warping $P P-P S$ (Figure 8), $P P-S H$ (Figure 9) and $P P-S V$ (Figure 10). More specifically, these three figures depict the extended $S$-wave impedance from $P P$ inversion (top left of the 3 figures), time shift (top right of the 3 figures). QC of time-shift (bottom left of the 3 figures).

One can see clearly (note the red crosses on the 3 figures) that a global constant vertical stretch (top left, A) is too rough to correctly align the $S$-wave impedances (bottom right, D). After applying a timeshift correction we can see much better alignment of the red crosses (bottom left $\mathbf{C}$ and right $\mathbf{D}$ images of the 3 figures)

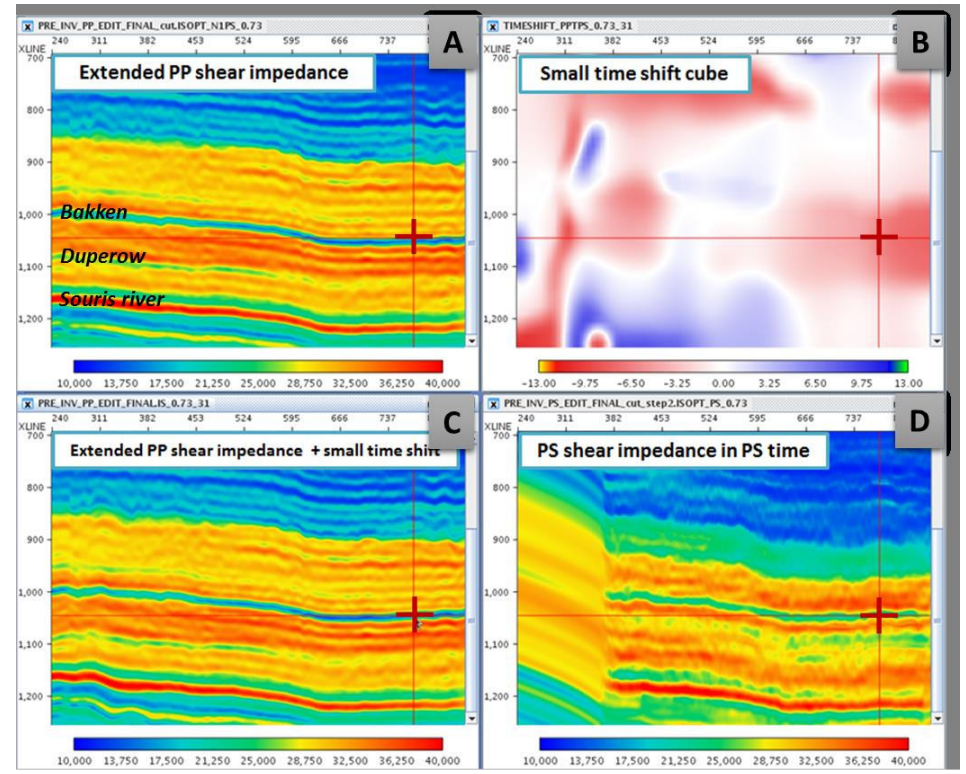

Figure 8: Inverted IS from PP-inversion after applying a constant global stretch (A, top left). This factor ${ }^{P P-P S}$ is obtained by identification of the interest area in $\mathrm{TWT}^{P P}$ and $\mathrm{TWT}^{P S}$ and is equal to (770$550) /(1100-800)=0.73$. Computation of PP-PS time shift $(B$, top right) resulting from the warping procedure. QC after applying time shift correction $(C$, left bottom). Red crosses at bottom are better aligned $(C$ and $D)$. 


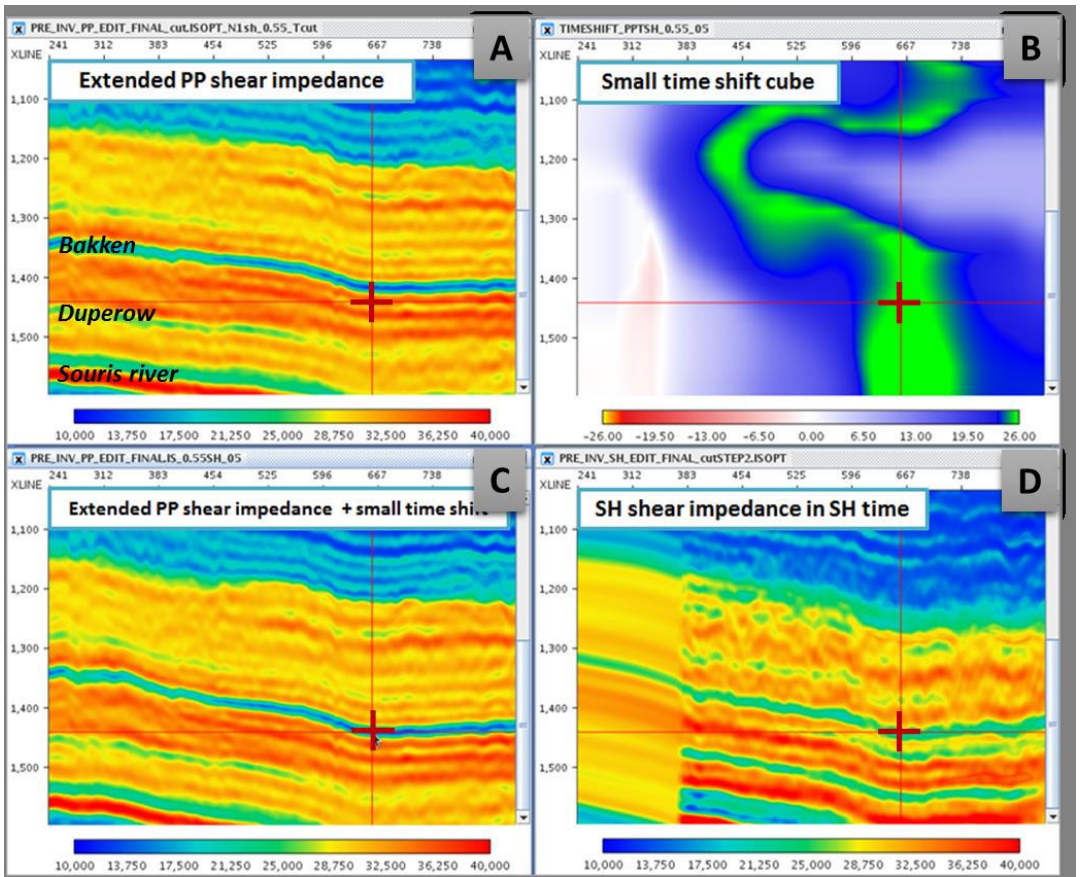

Figure 9: Inverted IS from PP-inversion after applying a constant global stretch (A, top left). This factor ${ }^{P P S H}$ is obtained by identification of the interest area in $\mathrm{TWT}^{P P}$ and $\mathrm{TWT}^{S H}$ and is equal to (770$550) /(1500-1100)=0.55$. Computation of PP-SH time shift $(B$, top right) resulting from the warping procedure. QC after applying time shift correction (C, left bottom). Red crosses at bottom are better aligned $(C$ and $D)$.

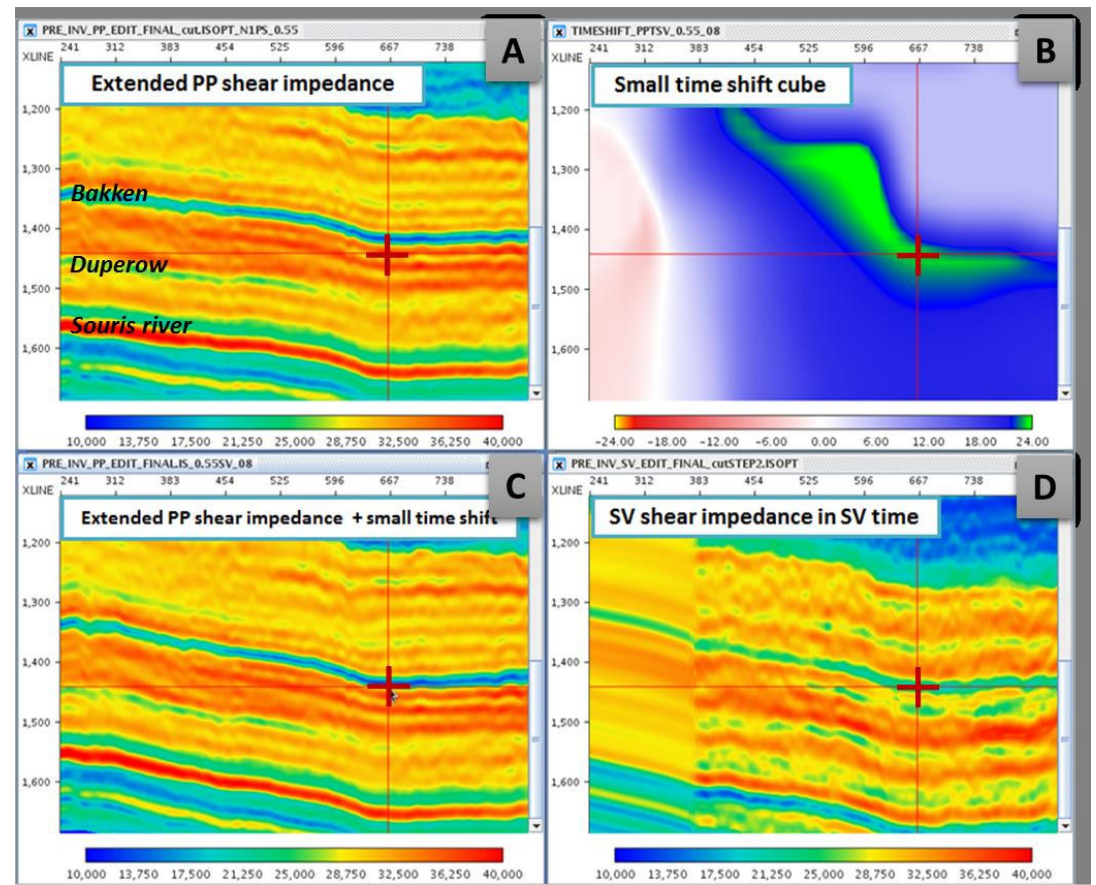

Figure 10: Inverted IS from PP-inversion after applying a constant global stretch (A, top left). This factor ${ }^{P P-S V}$ is obtained by identification of the interest area in $\mathrm{TWT}^{P P}$ and $\mathrm{TWT}^{S V}$ and is equal to (770$550) /(1500-1100)=0.55$. Computation of PP-SV time shift $(B$, top right) resulting from the warping procedure. QC after applying time shift correction $(C$, left bottom). Red crosses at bottom are better aligned $(C$ and $D)$.

\section{QC of multicomponent joint-inversion results (step 3) from Kevin Dome}


We usually perform two kinds of Quadri-joint inversion to QC the inversion process:

(1) the first one is done without taking into account the geological term of the cost function, in which the optimizer is not integrating any geological information according to mode of deposition and it is free to find the optimal solution without taking into account geological $a$ priori information. This is a useful first step because it reveals whether the optimizer is working well in a simple case (no constraints with geological information). For that reason we observed weaker residuals (Figure 11: right columns from the $P P, P S, S H, S V$ wavefields respectively, labeled as case NOGEOL). One can notice an exception for the middle angle class of $P S$ wavefield where residuals remain strong. This kind of QC could give clues to improve pre-processing of seismic amplitude before building of input angle stacks used by inversion.

The final inversion integrates two terms in the cost function: geologic and seismic and it is obviously more complex to optimize; we therefore expect stronger residuals compared to the first inversion. This behavior is indeed observed on Figure 11 with all the middle columns from 4 wavefields $(P P, P S, S H, S V)$ :
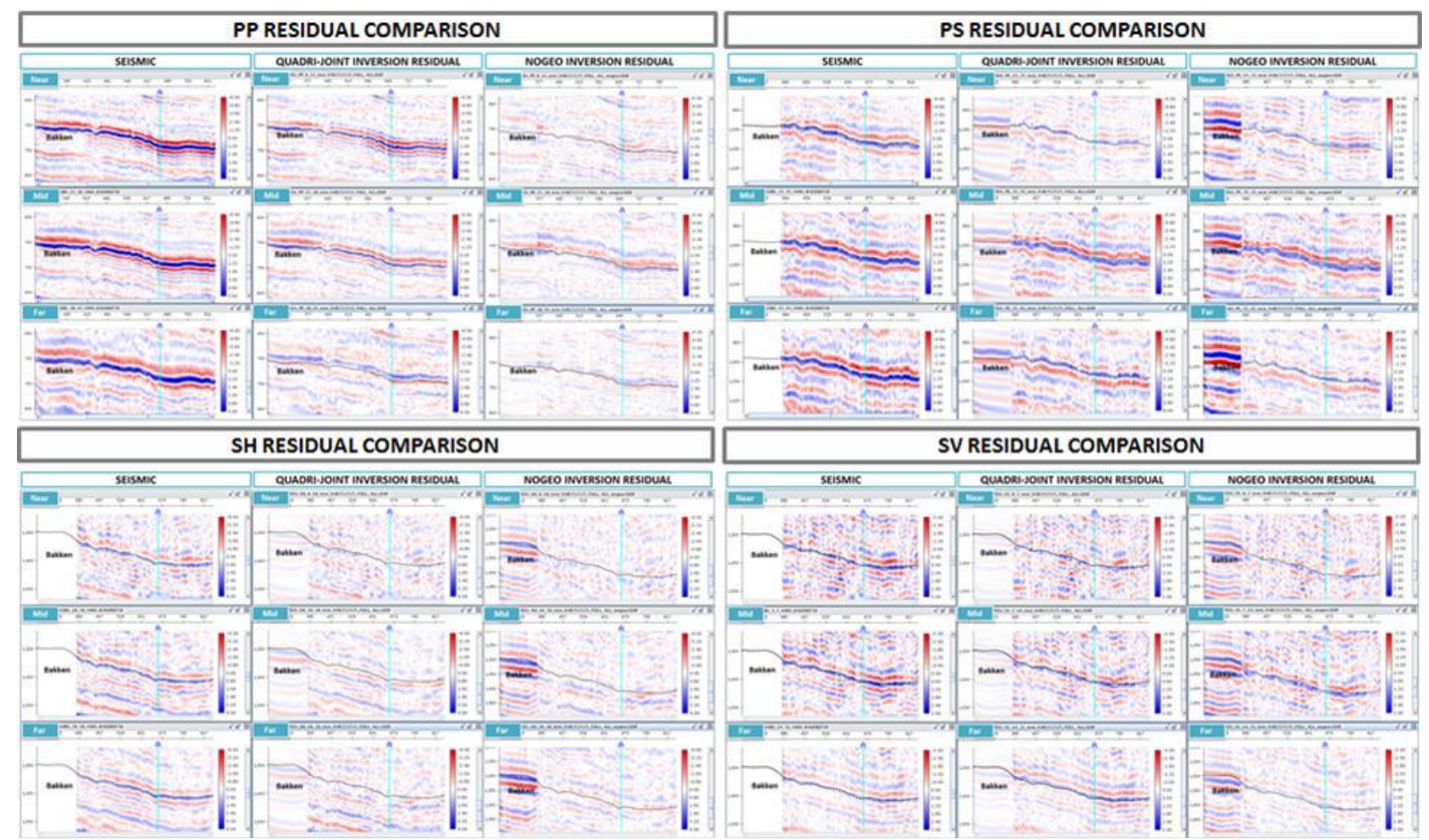

Figure 11: Comparison of residuals from two kinds of quadri-joint inversions, taking into account the geological term (middle columns for PP, PS, SH, SV wavefields) and without (right columns for PP, PS, SH, SV wavefields).

(2) The second QC consists of comparing the inverted log with real measured logs, as previously described in the text accompanying Figure 6 for the monocomponent inversion. Figure 12 illustrates the inverted log with final (including the geological and seismic terms) quadri-joint inversion (blue curves) and the measured log (red curves). 


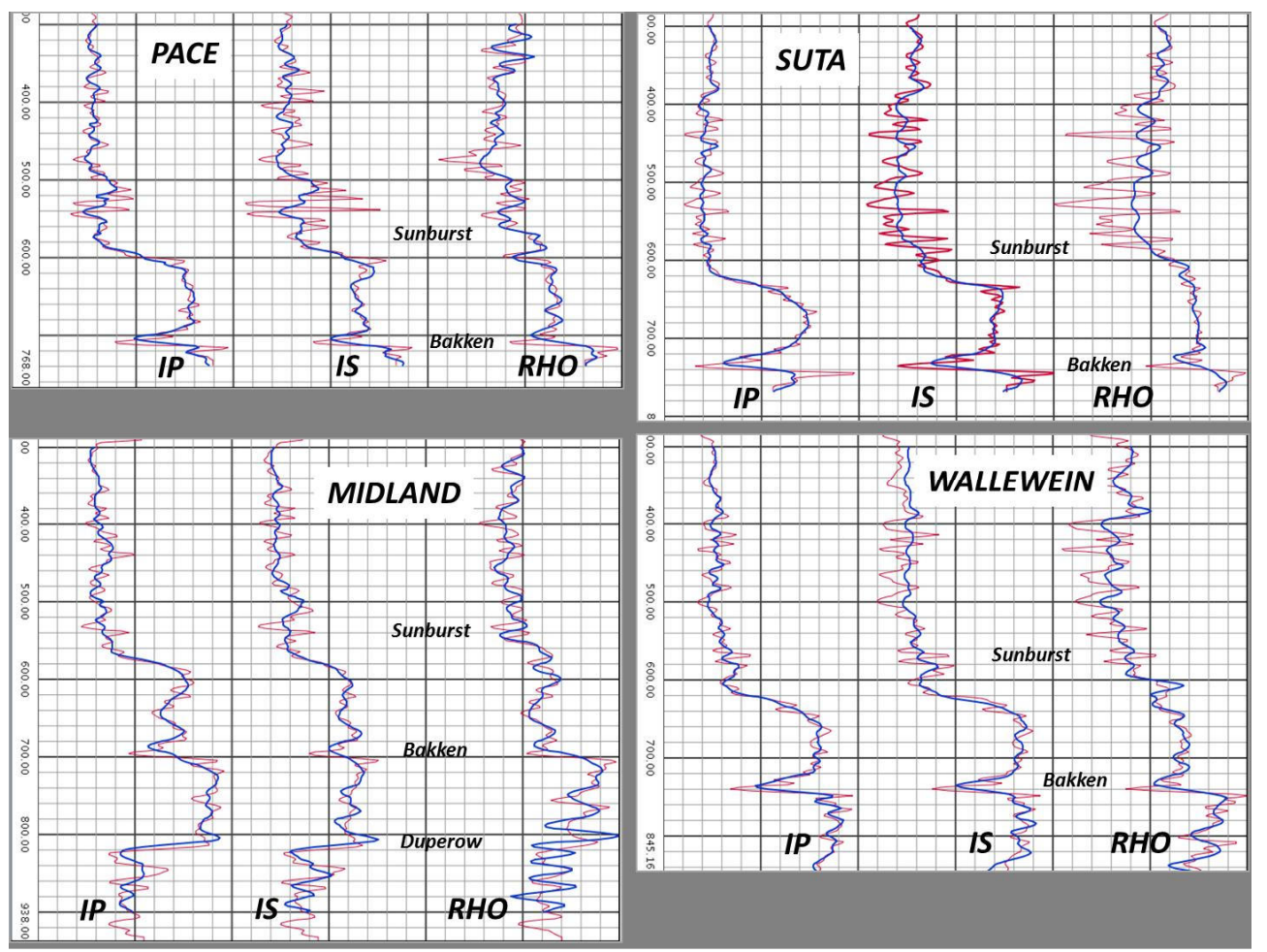

Figure 12: The oscillation-like ringing artefacts of the inverted density from PP inversion almost disappear from quadri-joint inversion (bleu curves), excepted from Midland 10 well which is very close to the edge of the multicomponent survey and for which shear acoustic log was simulated instead of being measured directly in the well.

\section{Application of quadri-joint inversion to subsurface characterization at Kevin Dome}

\section{Comparison of different kinds of joint-inversion results at the Duperow $\left(\mathrm{CO}_{2}\right.$ storage target $)$}

From the different kinds of jointly-inverted elastic parameters, we have extracted maps at the Duperow level to analyze the contribution of each wavefield (Figure 13). One can also notice the good similarities between the three tri-joint inversions; nevertheless the final quadri-joint inversion seems to be the best compromise between all previous joint inversions. Other QCs at wells (not shown here) also confirm the fidelity of quadri-joint inverted traces at wells compared to initial well logs filtered to the seismic bandwidth.

Looking at this result, one could conclude that jointly inverting the $P P$ and $P S$ data will be enough to provide the best result because density maps from bi-joint $\boldsymbol{P P}-\boldsymbol{P S}$ inversion are very similar to the quadri-joint inversion. In this regard, it is instructive to look at the density maps from the Bakken/Banff interval (Figure 16) and Sunburst (Figure 17). Looking at the Sunburst horizon, the bijoint $P P-S V$ inversion seems to provide a more similar result to the quadri-joint ( $P P-P S-S H-S V)$ inversion. Looking at the Bakken formation, however, only the tri-joint PP-SV-PS inversion looks similar to the quadri-joint $P P-P S-S H-S V$ inversion. 


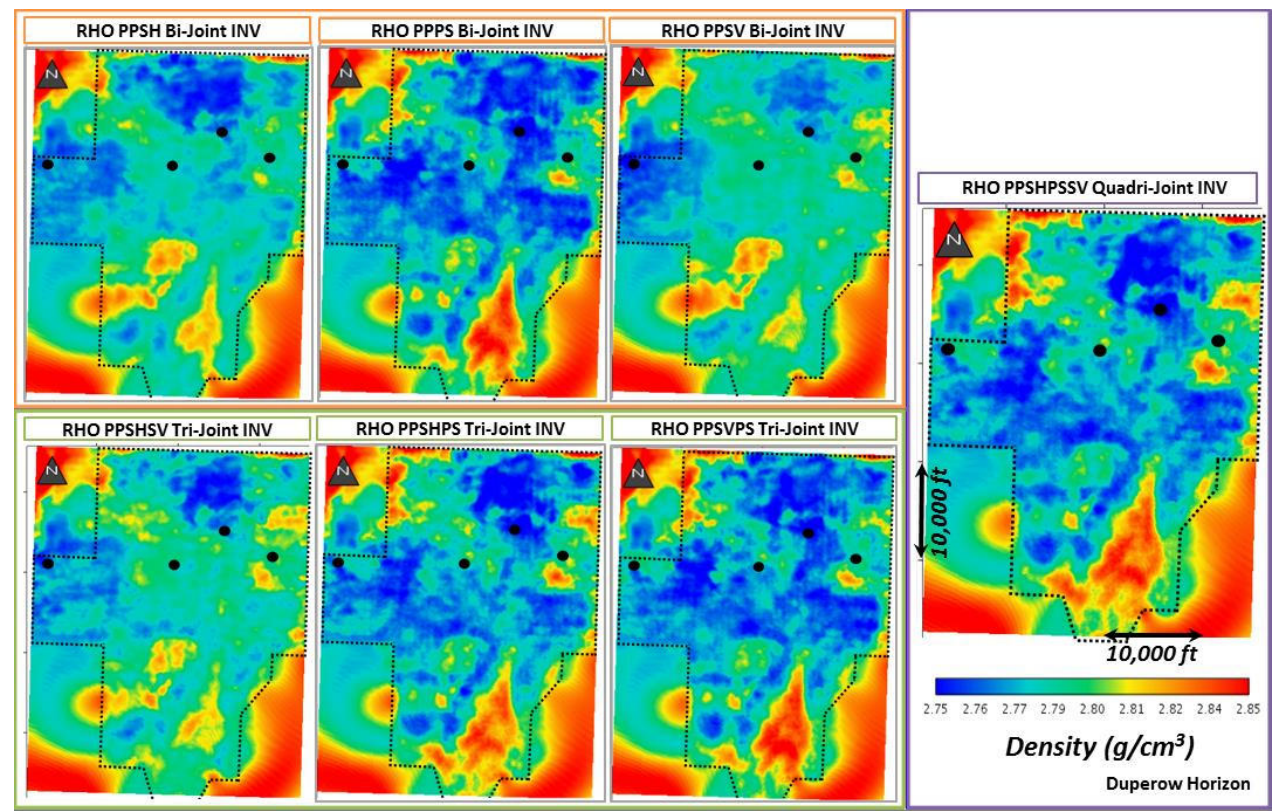

Figure 13: Comparison at mid-Duperow horizon of the inverted density parameter obtained with different kinds of wavefields. Bi-joint inversion (3 images at the top), Tri-joint (3 images at the bottom) and quadri-joint inversion (right). Black dots mark well locations used for the joint inversion study. bi-joint PP-PS inversion is very similar to the final quadri-joint PP-PS-SH-SV inversion (right).

\section{Comparison of different kinds of joint-inversion results at the Bakken/Banff interval (unconventional target)}

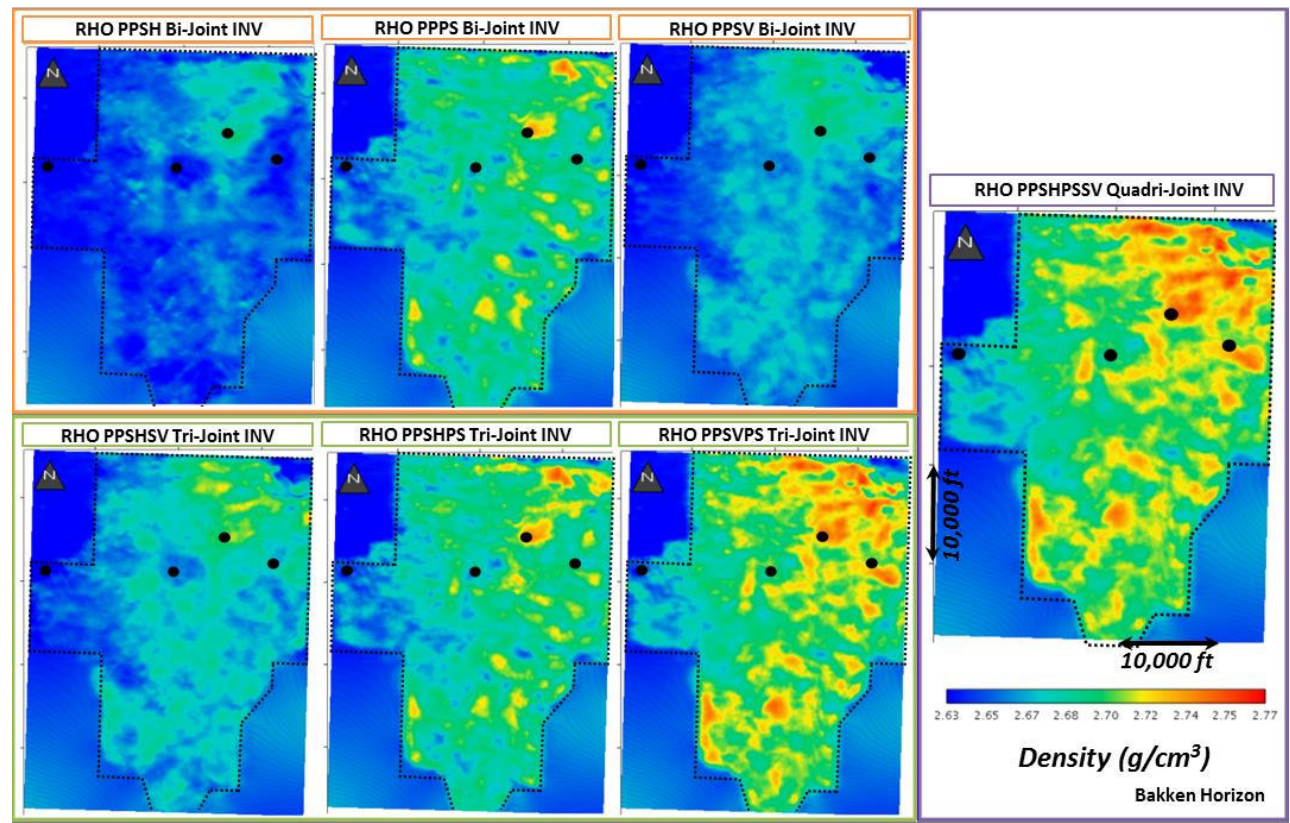

Figure 14: Comparison at Bakken horizon of inverted parameter density obtained with different kinds of wavefields. Bi-joint inversion ( 3 images at the top), Tri-joint (3 images at the bottom) and quadrijoint inversion (right). Black dots mark well locations used for the joint inversion study. Tri-joint PP$S V-P S$ inversion looks similar to the final quadri-joint PP-PS-SH-SV inversion (right), this density map has to be compared with the one displayed on the right map of the Figure 7 (density from PP inversion)

\section{Comparison of different kinds of joint-inversion results at Sunburst (conventional target)}




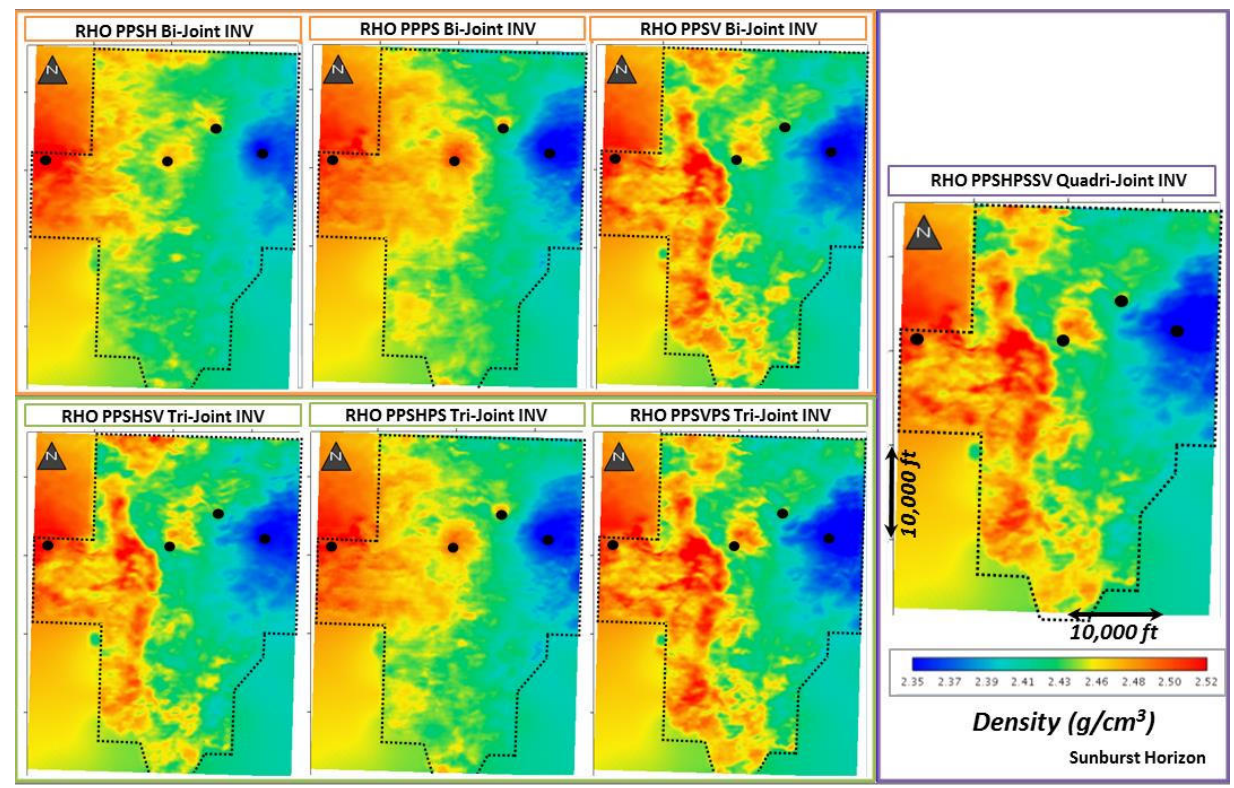

Figure 15: Comparison at Sunburst horizon of inverted density parameter obtained with different kinds of wavefields. Bi-joint inversion (3 images at the top), Tri-joint ( 3 images at the bottom) and quadri-joint inversion (right). Black dots mark well locations used for the joint inversion study. Bijoint PP-SV inversion seems to provide the more similar result to the final quadri-joint PP-PS-SH-SV inversion (right).

\section{Comparison with well data: mid-Duperow porosity zone}

Of the three wells that penetrate the mid-Duperow porosity zone in the 9C 3D survey area only the two recently drilled by the BSCSP acquired modern wireline shear data to permit quantitative comparison of all of the inversion results with the wells. The other well contained density and sonic $\operatorname{logs}$ which permit comparison of the estimated $P$-wave impedance $(I P)$ and porosity with the inversion results. Despite the relative paucity of well control, average properties within the midDuperow porosity zone were computed and compared with the inversion results for each of the main outputs from the quadri-joint inversion.

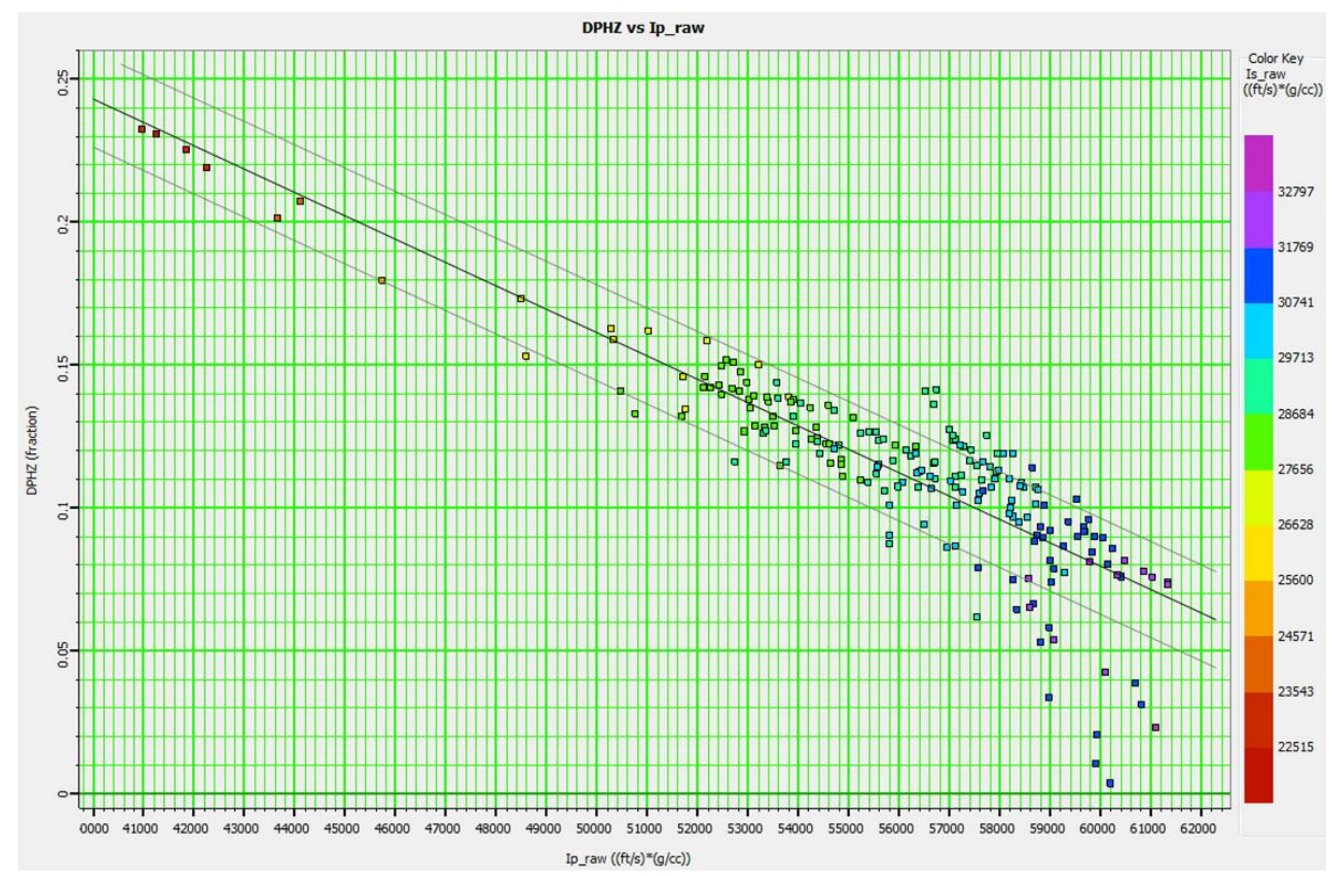


Figure 16: Crossplot between density porosity and computed $P$ impedance (IP) from the Wallewein 22-1 well over the Middle Duperow porosity interval. Note the very good correlation observed between the two quantities. The correlation coefficient between the two quantities is 0.87. Colored values are measured IS values.

\section{Mid-Duperow porosity estimation from rock physics transforms: comparison with direct density estimation}

Crossplotting IP derived from the sonic log in the Wallewein 22-1 well drilled by the Project with measured porosity (computed on a dolomite matrix from the density log), as shown in Figure 16 reveals an excellent correlation between $I P$ and the porosity in the Middle Duperow porosity zone that is the primary target for sequestration in this study. A similar result with slightly better correlation is observed in Figure 17 for $I S$ measured in the Project's wells.

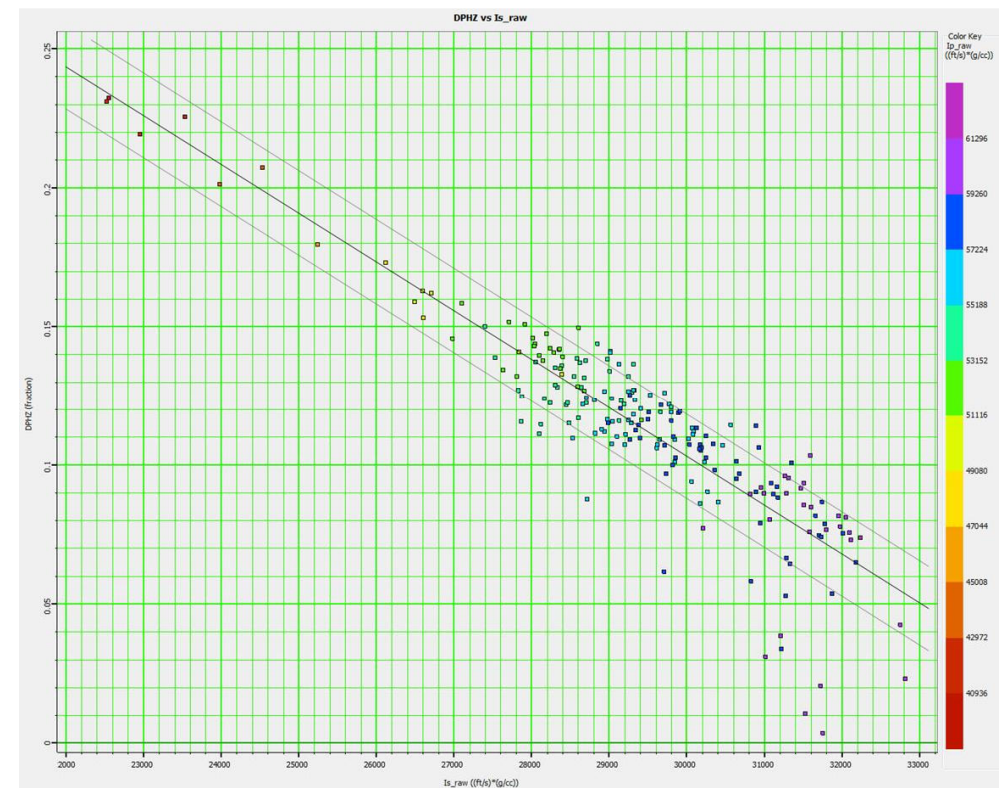

Figure 17: Crossplot of measured density porosity and shear impedance (IS) in Wallewein 22-1 well in mid-Duperow porosity zone. Note excellent agreement between measured two quantities with correlation coefficient of $\mathbf{0 . 8 9}$. Colored values are measured IP values.

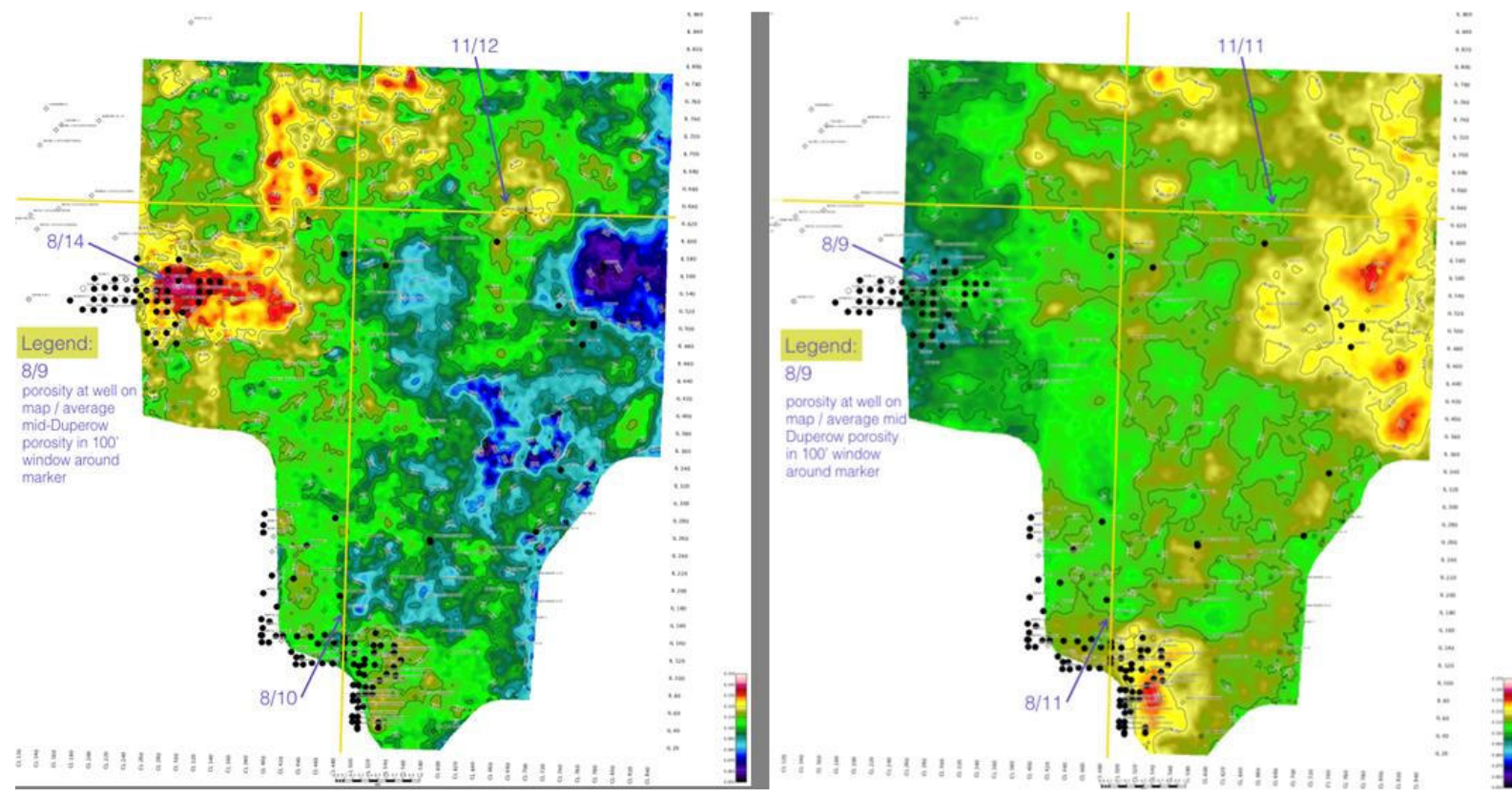


Figure 18: Transforms derived from porosity-impedance regressions using IS (left) and IP (right) maps for the Middle Duperow porosity zone with well locations annotated and well-derived values for porosity annotated with values derived from each map at well locations.

These results provide encouragement for generating transforms using the regression equations in both Figures to yield seismically-derived estimates of porosity in the Middle Duperow zone. The results of these transforms applied to the average mid-Duperow IP and IS extractions from the quadri-joint inversion are shown in Figures 18 and 19.

Generally, the porosity estimate derived from the P impedance transform (Figure 18) had both the best absolute agreement with the average porosity in each of the three wells and the most geologicallyreasonable appearance given the .
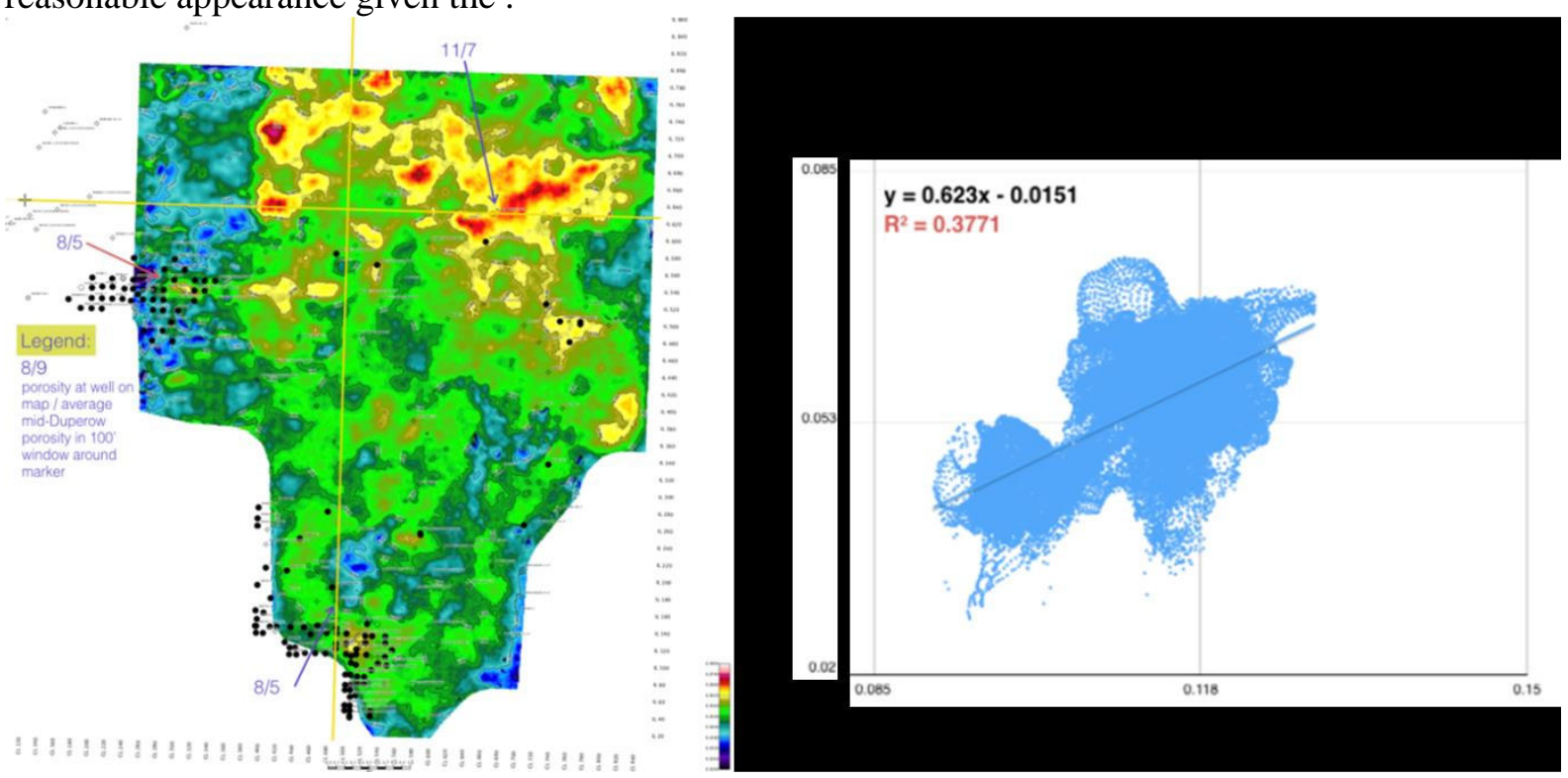

Figure 19: Mid-Duperow porosity derived from average density values from quadri-joint inversion converted to porosity using a dolomite matrix (left) and cross plot of this map with values derived from IP-based regression shown in Figure 18. Note the poorer agreement with well values for the density-derived estimate but generally smaller amount of absolute variation than for that derived from the IP regression.

The $S$-wave impedance-(IS) derived porosity transform generally had reasonable agreement with the values observed in the wells except at the Midland 10 well located on the far western edge of the survey. A possible explanation for the discrepancy is an erroneous background model in this area caused by the use of estimated shear slownesses in that well because no measured shear was recorded in the Midland 10 well, necessitating an estimation of shear slownesses using the other two wells as training data.

The porosity derived from applying a dolomite matrix value to the inverted density output (Figure 19) shows decent although not excellent agreement with the values derived from the IP transform, as seen in the cross plot of Figure 19. Given the higher noise level inherent in the density estimate (even from an inversion utilizing pure shear angle stacks) compared to that of the IP estimate (cf. Swan, 1993), the observed agreement appears reasonable. Additionally, although the density-derived porosity's absolute tie to the average porosity at each well is inferior to that derived from IP, the former exhibits less interwell variation, which is more geologically reasonable than the other two maps which exhibit variation that is probably too large in the relatively low-porosity environment characteristic of Devonian stromatoporoid buildups such as the mid-Duperow here.

\section{Further comparison of inversion results with Bakken and Banff horizon maps}


In this part we focus our attention on the Bakken and Banff formations because they exhibit less lateral variation of rock properties and fluid content and we can evaluate more easily the differences from independent inversion of each wavefield (Figures 20, left and middle columns). We have also extracted Bakken horizon maps from these inversion results considering the three elastic parameters $I P, I S$ and Density. On Figure 20 (top row) we have displayed them at a different scale to better see the distribution compared to the final quadri-joint inversion result. We can observe similar trends with $I P$ (top row) and IS (middle row) and for both a drastic reduction of inverted noise (no acquisition footprints are visible from quadri-joint inversion, right column), but the ranges of values are quite different. For the density result there is no trend at all between single inversions and the range of values is quite different (bottom row).

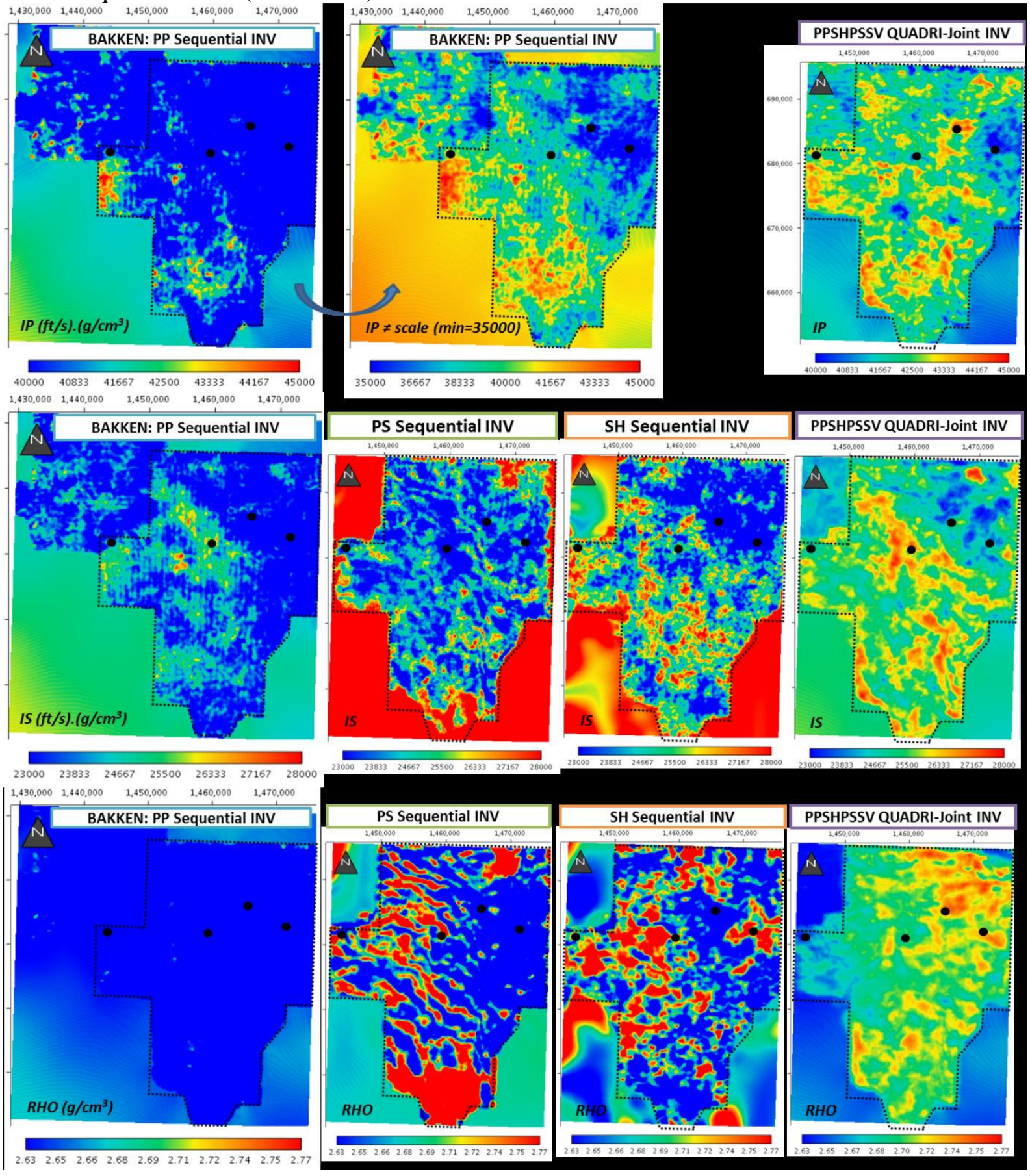

Figure 20: At top row, P-wave impedances (IP) from PP-inversion compared to quadri-joint inversion.. We can observe a similar trend of color in the south west and north-east (middle image with different scale view. At middle row, $S$-wave impedances (IS) from PP-inversion, PS-inversion, SH- 
inversion compared to quadri-joint inversion. We can observe a general trend from north-west to south-east with PS-, SH-, and quadri-joint inversion. Also distribution of colors are quite different from all inversion. At bottom row, density from PP-inversion, PS-inversion, SH inversion compared to quadri-joint inversion. We observe a quite different trend of color between all single inversion results. One can notice the quadri-joint inversion result (right column) shows a reasonable range of values compared to the left and middle columns while the PP result has values that are all well below the correct values.

It is important to note that the $P P$ wavefield is usually the only attribute available to generate the combination of $P$ - and $S$-wave inverted impedances (IP and $I S)$ used to deduce the brittleness of rock (e.g. Chopra and Sharma, 2015, Sharma and Chopra, 2015a). The previous comparisons demonstrate that the quadri-joint inversion result gives the best estimate of $I P$ and $I S$ with which to compute geomechanical attributes like Young's modulus and Poisson's ratio, directly from all three inverted elastic parameters $I P, I S$ and density.

We can compare geomechanical attributes derived solely from the from $P P$ data (Figure 7 , middle row) and quadri-joint (PP-PS-SH-SV) pre-stack inversion (Figure 20, right column). We focus on the Bakken horizon map for the reasons previously discussed, to wit that we do not expect strong variations of rock properties in this formation. The results are displayed below in Figure 21. We observe at the top row of Figure 21 a wider distribution of values compared to the bottom row for both geomechanical attributes. The acquisition footprint is also more visible (Figure 21, top right) for the Young's modulus estimate $(\boldsymbol{E})$. These geomechanical attributes deduced from seismic inversions follow the same trend but from the quantitative point of view differences are great between usual $P P$ inversion and quadri-joint inversion. Geomechanical values from quadri-joint inversion have a smaller range of values which is far more reasonable given the very small inter-well variation observed in the wells that penetrated the Bakken/Banff interval.

While it would be possible to limit the range of values observed on the $P P$-only inversion by reducing the value of the uncertainty-density parameter, for example $\sigma_{\rho}=0.1$. Of course in this case we will not have access to more detailed density distribution compared to the $a$ priori density model. We remind it is built by extrapolation of well log information according to the mode of deposition inside each geological units. It is important to note that we did not do that in this case and the results compared in Figure 20 were all generated using identical values of inversion parameters. 


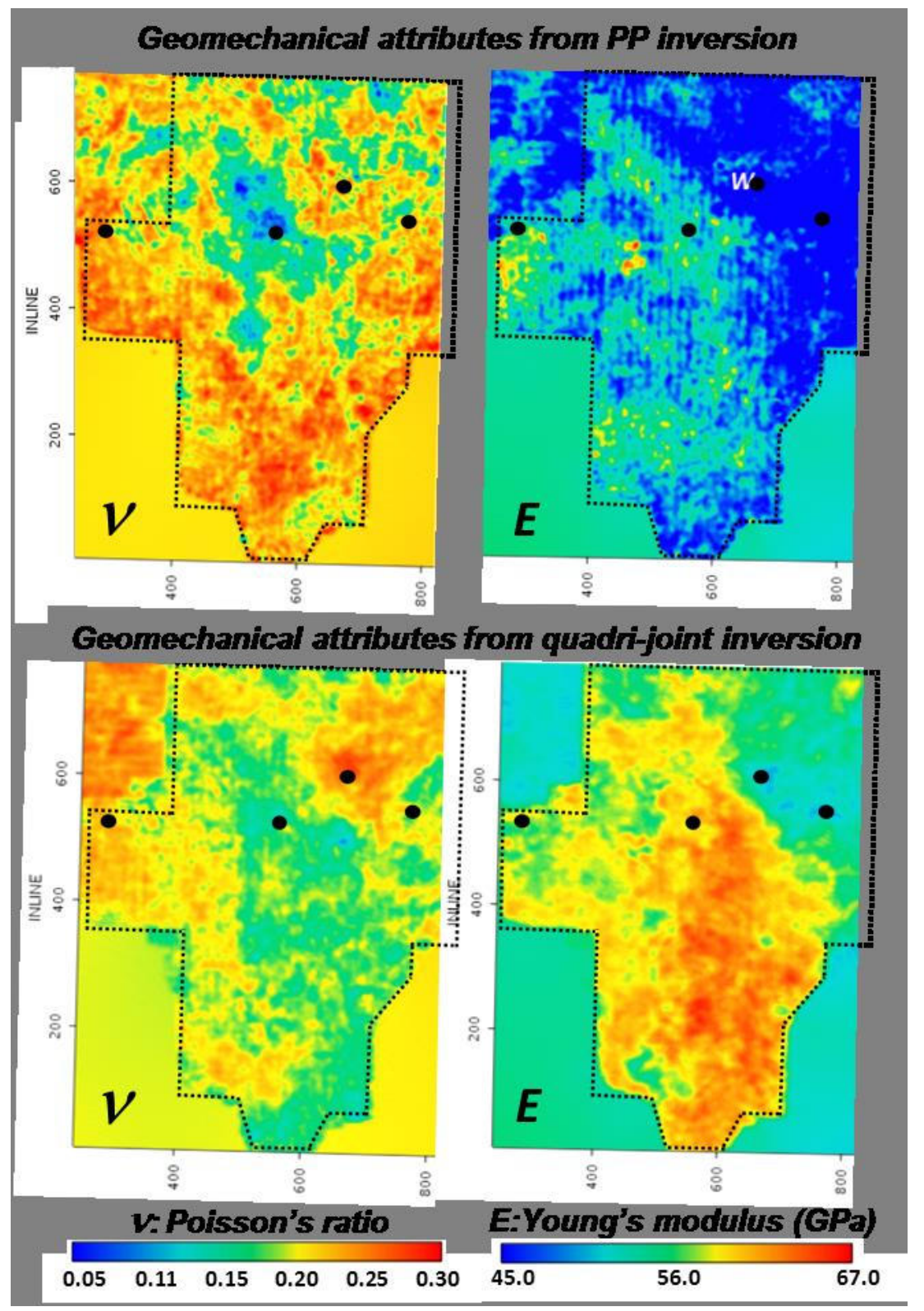

Figure 21: Geomechanical parameters computed at the Bakken/Banff horizon map. They are computed from inverted $P$ - and $S$-wave impedances and Density obtained with PP (top) and with final quadri-joint (PP-PS-SH-SV) pre-stack inversion (bottom). One can notice acquisition footprint appearing with PP-inversion only (top row) and Young's modulus is more affected (E) than Poisson's ratio (n). A larger range of geomechanical attribute values is also more important with PP-inversion results (top row), but are less credible from inversion QCs (Figure 6).

It is interesting to compare the geomechanical attributes deduced from seismic inversion with laboratory measurements of the middle Bakken formation from the adjacent Williston Basin. Given the great disparity in resolution between seismic data and high-frequency laboratory experiments on core samples, good agreement between the two sets of measurements is far from assured. In addition to the different resolutions, one has to keep in mind the complex intermediate steps required to generate the seismic estimates of the elastic properties, which include recording and pre-processing of multicomponent seismic and the final stratigraphic inversion, which itself depends on proper multicomponent seismic imaging to build angle stacks for each wavefields. 
Figure 22 is a cross plot of laboratory measurements (Pei et al., 2016) of Poisson's ratio with Young's modulus. Each blue point comes from laboratory measurements. Values cluster between Poisson ratios in the range of $[0.15 ; 0.25]$ and Young's moduli of $[50 ; 70 \mathrm{GPa}]$. Considering $P P$ - and quadri-joint inversions (Figure 17), we have delimited extremum values by ellipses. The red ellipse corresponds to geomechanical parameters deduced from the usual $P P$-inversion and the green one is the quadri-joint inversion result.

From Figure 22 it is apparent that the smaller green ellipse seems to focus better on the stronger density of blue points. This would seem to indicate that the quadri-joint inversion follows the same trend of laboratory measurement from Middle Bakken formation. Further investigations will be necessary to explain blue points for which which Young's modulus is greater than $80 \mathrm{GPa}$.

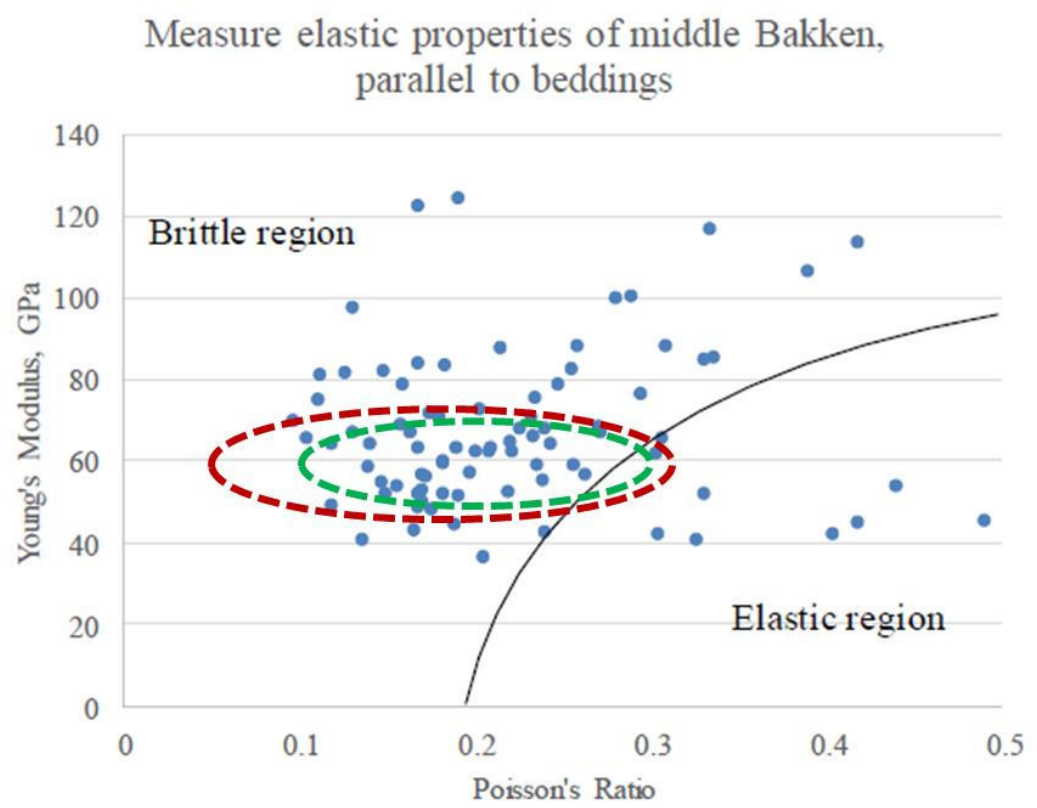

Figure 22: Laboratory measurements of elastic rock-properties from middle Bakken (parallel to beddings) are identified by blue points (Pei et al., 2016). Then extremum values of geomechanical attribute (Figure 21) deduced from the PP-inversion and quadri-joint inversion respectively are demarcated by red and green ellipses. Both ellipses are more or less centered and we clearly see the green ellipse surrounding a strong density of blue points.

\section{Conclusions}

We have applied a multicomponent pre-stack inversion workflow on a real case study recorded at Kevin Dome in Montana. The workflow has successfully handled twelve different seismic cubes corresponding to three angle stacks (usually called Near, Mid and Far) for the $P P, P S, S H$ and $S V$ wavefields and is the first published example of which we are aware that has successfully inverted both pure shear modes with converted-wave data against a single, common earth model. To perform the inversion, we have created registration laws through a novel 3D warping procedure working with inverted $S$-wave impedances. These registration laws are necessary to run different kinds of joint inversions like bi-, tri- and quadri-joint inversions.

After doing all necessary QCs including residuals compared to the original input seismic data and trace extractions from inverted cubes at four well locations, we have shown the added value of jointly inverting all available multicomponent wavefields. Examination of horizon maps at the three main targets of interest in the survey, namely the Sunburst (conventional), Bakken/Banff (unconventional) 
and Duperow $\left(\mathrm{CO}_{2}\right.$ storage), the added value of the final quadri- joint inversion of $P P, P S, S H$ and $S V$ wavefields is readily apparent.

The resulting impedance and density estimates from the quadri-joint inversion combine reflection coefficient information from all modes employed and therefore produce a more accurate estimate of each of the three isotropic parameter combinations resolvable by wide-angle surface seismic data than would be possible using any single mode. In particular, the density attribute is much more robustly recovered than would be the case using $P P$ or $P S$ data only.

Special attention was given to comparing results among the various input datasets over the Bakken/Banff interval because this interval exhibits much less lateral variation than the other targets and is also a resource play objective both in the Alberta Basin immediately north of the study area and also in the Williston Basin to the east of the study area. We observe good agreement between Young's Modulus and Poisson's Ratio derived from the quadri-joint inversion and published laboratory results from the Middle Bakken formation in the adjacent Williston Basin. To obtain a usable Young's Modulus, the inverted density is a crucial parameter that cannot be recovered in a usable fashion from $P P$-inversion only given its extremely low signal-to-noise ratio for that mode. The geomechanical parameters deduced from quadri-joint inversion are clearly superior to those derived from $P P$-only results when compared to laboratory measurements.

Additionally, by registering a single, common physical quantity-IS-derived from each wavefield, our multicomponent pre-stack inversion workflow represents a significant improvement over methods which rely on attempting to match trace waveforms which may have different phase, frequency content, and polarity.

Finally the multicomponent pre-stack stratigraphic inversion is an elegant way of employing all modes of a multicomponent acquisition to generate an optimal estimate of all three elastic parameter $P$ - and $S$-wave impedances and density. Further study will be necessary to use the inverted density to discriminate lithology and fluid contents and also to use the geomechanical parameters coming from inversion as a predictive tool in the context of resource play sweet spot characterization.

\section{Acknowledgements}

The Big Sky 9C 3D seismic survey was acquired by the Big Sky Carbon Sequestration Partnership, funded under DOE Award Number DE-FC26-05NT42587. The authors would like to specifically thank Lee Spangler, the director of BSCSP, for his help and persistence. 


\section{References}

Agullo, Y., Macé, D., Labat, K., Tonellot, T., Bourgeois, A., and Marc Lavielle [2004], Joint PP and PS stratigraphic inversion for prestack time migrated multicomponent data. $74^{\text {th }}$ SEG Annual Meeting, Denver. Expanded Abstracts, 889-892.

Aki, K., and Richards, P.G., [1980], Quantitative seismology: Theory and methods, Vol.1: W.H. Freeman and Company, New York. ISBN 0716710587.

Barnola, A., S. and Ibram, M., [2013], 3D simultaneous joint PP-PS prestack seismic inversion at Schiehallion field, United Kingdom Continental Shelf. Geophysical Prospecting, 62, 278-292.

Butler, E., Mueller, S. and Davis, T.L., [2016], Application of time-lapse multi-components seismic inversion to characterize pressure and stimulation in the Niobrara and Codell Reservoirs, Wattenberg Field, Colorado. First Break, vol.34.

Chopra, S., and Sharma, R.K., [2015], Determining Brittleness from Seismic Data, Search and Discovery Article \#41725.

Dariu, H., Garotta, R., and Granger, P.,Y., [2003], Simultaneous inversion of PP and PS wave AVO/AVA data using simulated annealing. $73^{\text {rd }}$ SEG Annual International Meeting, Expanded Abstracts, 22, 120-123.

DeVault, B., Tonellot, T., Macé, D., Ker, S., Pichard, M., [2007], Prestack 9-C joint inversion for stratigraphic prediction in the Williston Basin. $77^{\text {th }}$ SEG Annual International Meeting, San Antonio, Expanded Abstracts, 1039-1043.

Garotta, R., Granger, P.Y., Dariu, H., [2000], Elastic parameter derivations from multi-component data. $70^{\text {th }}$ SEG Annual International Meeting, Expanded Abstracts.

Garotta, R., Granger, P.Y., Dariu, H., [2002], Combined interpretation of PP and PS data provides direct access to elastic rock properties. Leading edge, 532-535

Hilterman, F.J., [2001]. Seismic Amplitude Interpretation. (Distinguished Instructor Series SEG/EAGE, No 4)

Lebrun, D., Richard, V., Mace, D., and Cuer, M., [2001], SVD for multi-offset linearized inversion. Resolution analysis in multicomponent acquisition: Geophysics, 66, 871-882.

Murray, P.E., Popov, G., and DeVault, B.C., [2016], Orientation of multicomponent receivers via semblance analysis. SEG International Exposition and 8 $7^{\text {th }}$ Annual Meeting, Dallas, p.2253-2257.

Pei, P., Ling, K., Hou, X., Nordeng, S., Johnson, S., [2016], Brittleness investigation of producing units in Three Forks and bakken formations, Williston basin. Journal of Natural Gas Science and Engineering, 32, 512-520.

Ramos, A.C.B., Castagna, J.P., [2001], Useful approximations for converted-wave AVO. Geophysics, $66,1721-1734$.

Rüger, A. , [1996]. Reflection coefficients and azimuthal AVO analysis in anisotropic media.

Sharma, K.R., and Chopra, S., [2015a], New attribute for determination of lithology and brittleness of rocks with a new attribute. The Leading Edge, 34, 554-564. 
Sharma, K.R., and Chopra, S., [2015b], Identification of thin sweet spots in the Duvernay Formation of north central Alberta. SEG International Exhibition and 86th Annual Meeting, New Orleans, 18021806.

Side, J., Cambois, G., and Vuillermoz, C., [2000], Shear-wave velocity and density estimation from PSwave AVO analysis: Application to an OBS dataset from the North Sea. GEOPHYSICS, SeptemberOctober 2000, Vol. 65, No. 5 : pp. 1446-1454. https://doi.org/10.1190/1.1444833

Swan, H.W., [1993], Properties of direct AVO hydrocarbon indicators in Castagna, J. P., and Backus, M. M., Eds., Offset dependent reflectivity--Theory and practice of AVO analysis: Society of Exploration Geophysics, 78-92.

Tonellot, T., Macé, D., Richard, V., and Cuer, M., [2001], Joint stratigraphic inversion of anglelimited stacks. $71^{\text {st }}$ SEG Annual International Meeting, San Antonio, Expanded Abstracts, 227-230. 